\title{
Recent Research and Development Status of Relaxed Optics and Laser Technology: A Review
}

\author{
Petro Trokhimchuck \\ Anatolii Svidzinskyi Department of Theoretical and Computer Physics, Lesya Ukrainka Volyn' National University, Lutsk, Ukraine \\ Email: trope1650@gmail.com
}

How to cite this paper: Trokhimchuck, $\mathrm{P}$. (2021) Recent Research and Development Status of Relaxed Optics and Laser Technology: A Review. Optics and Photonics Journal, 11, 210-263. https://doi.org/10.4236/opj.2021.117015

Received: May 13, 2021

Accepted: July 25, 2021

Published: July 28, 2021

Copyright (c) 2021 by author(s) and Scientific Research Publishing Inc. This work is licensed under the Creative Commons Attribution International License (CC BY 4.0).

http://creativecommons.org/licenses/by/4.0/

\begin{abstract}
It is shown that for laser technologies it was necessary to create a new branch of physics: Relaxed Optics (synthesis of methods of the physical optics, quantum electronics, physical chemistry, physics of irreversible phenomena in unitary system). It is allowed to explain complex chain processes of interaction light and matter. Possible applications of Relaxed Optical methods for the modeling of the laser-induced processes phenomena, including laser implantation (surface and subsurface processes), laser-induced optical breakdown (volume processes) and laser annealing of radiation and other defects in solid, are discussed. Perspectives of using these methods for the creation of new laser technologies, including creation new types of optoelectronic devices (heterostructures, diffraction lattices, etc.), resolution the problems of metallurgy, material science, painting, architecture and a building, are analyzed.
\end{abstract}

\section{Keywords}

Laser Technology, Relaxed Optics, Laser-Induced Processes, Laser

Implantation, Heterostructures, Cascade Models, Structural Coherence

\section{Introduction}

Relaxed Optics (RO) is the chapter of modern physics, which is created for the description of processes of the second-order relaxation in the first-order optical excited states [1]. We show that basic chapters of modern optics, including linear and nonlinear may be explained with this point of view, too, as processes of radiated relaxation [2] [3]. Second part is the processes of non-radiated relaxation [1] [2]. These processes are connected with phase transformations of irradiated matter [1] [3]. Therefore, RO may be represented as physics of laser technology, too [1] [3].

But RO may be represented as fuller realization of the famous Newtonian 
phrase: "The transitions of bodies into light and light into bodies obey the laws of Nature, which, as it seems, amuses itself with these transformations" [4] [5]. Relaxed Optics makes it possible to combine the processes of radiative and nonradiative relaxation into a single system and thus to consider more complex cascade processes that are characteristic of laser technology [1] [6] [7].

Roughly speaking RO is synthesis of Linear and Nonlinear Optics, photochemistry, radiation physics of status solid, physics of phase transitions and transformations, physical chemistry in one system. Therefore, the concept of structural coherence as third concept of RO was created and developed, too [1].

Main experimental data (laser-induced generation of surface and volume structures, laser annealing of radiation defects) and its explanation as Relaxed Optical phenomena are represented.

These processes are of different nature and various modeling methods are used to describe them. For surface processes, a cascade model of excitation of the corresponding type of chemical bonds in the saturation mode of radiation excitation was used [1] [7]. In this case, the processes of re-radiation and re-absorption of radiation play an important role. The main role in this is played by the intrinsic absorption of radiation.

Bulk processes are represented by laser-induced optical breakdown of the medium and the formation of filaments. Here, the main role is played by the processes of transformation of weakly absorbed radiation and the medium (diffraction stratification, generation of Cherenkov radiation and interference of this radiation), which ultimately leads to breakdown of the medium [7] [8] [9]. Also, for relatively short irradiation modes, the main ones are electromagnetic (ionization) shock processes, which can be accompanied by the release of heat.

Some peculiarities of laser technology and using the methods of RO for its receiving are discussed, too; Among them are laser annealing of radiation damages, creation of $n-p$ and $p-n$ junctions, creation of new types of heterostructures, increasing the resource of the corresponding elements of optoelectronic systems, painting, architecture, metallurgy and material science [1] [7].

Absorption mechanisms (at stable or unstable or metastable centers) play an important role in laser technologies. So, for annealing radiation defects, we must choose the radiation that is absorbed by these defects; in painting, radiation, which allows you to restore the original structure of paints. The temporal processes of irradiation do not have a great influence. In laser implantation of solids, the processes of saturation of radiation and the irradiation time play an important role [1] [8] [9].

\section{Main Experimental Data of Relaxed Optics and Its Explanation}

\subsection{Surface Processes and Phenomena}

Main experimental data of RO may be caused by the mechanisms of absorption (scattering) of light (on stable or unstable centers). In the first case, dynamic sa- 
turation of excitation plays an important role, in the second, the exposure time does not play a large role, the main thing is the integral radiation dose [1].

Therefore, we represent three types of RO experimental data. First is surface laser-induced processes [8] [9]. Here we focus on the laser-induced generation of cascade structural phase transformations of the irradiated material (the formation of interferograms and hedgehog-like structures). These structures are created after laser irradiation germanium and silicon with various regimes of irradiation. In this case, we have light scattering on stable centers. Such structures are obtained by irradiation with a series of pulses, both nanosecond and femtosecond. This avoids heating of the irradiated material.

Among the poorly studied problems in modern physics, the problem of self-absorption, especially in the regime of saturation of excitation, stands apart. In this case, we must pass from the concepts of the band theory of solids to the physical-chemical concepts. Even H. Haken pointed out the analogy between nonlinear optical phenomena and the theory of second-order phase transitions [10]. If we take into account that chemical reactions can also be attributed to phase transformations, then naturally the results of stepwise multiphoton ionization of chemical bonds in crystals can be attributed to both first-order phase transitions and polytropic transformations. The difference from nonlinear optical phenomena here is the secondary relaxation of primary excitations: for phase transformations it must be nonradiative. According M. Lax processes of nonadiabatic processes for light absorption are beginning for $h v>0.95 E_{g}$ [11] [12].

Detail researches of creation of the surface laser-induced structures are represented by A. Medvid' in [13]. Samples of Ge $\{111\}$ and Ge $\{001\}$ i-type single crystals are using in experiment. Nd:YAG laser (wavelength $1.064 \mu \mathrm{m}$, duration of pulse $15 \mathrm{~ns}$, pulse rate $12.5 \mathrm{~Hz}$, power $P=1 \mathrm{MW}$ ) was used for the irradiation. The AFM picture of Ge surface after Nd laser irradiation is representing in Figure 1 [13].

The more large height of nanohills is caused of processes the creation new phases (hexagonals). Initial structures had cubic (diamond) symmetry [13]. Medvid explained these results on the basis Benard phenomena [13]. But these results may be explained with cascade model of excitation of proper chemical bonds in the regime of saturation the excitation [1] [8] [9].

Laser-induced phase transformations are possible for silicon, too.

More large columns (height $20 \mu \mathrm{m}$, diameter 2 - $3 \mu \mathrm{m}$ ) were received after irradiation of pulse series the nanosecond eximer KrF-laser (wavelength $248 \mathrm{~nm}$, pulse duration $25 \mathrm{~ns}$ ) (Figure 2(a)) [14]. This figure illustrates the high aspect ratio silicon micro-columns that were formed in air after 1000 laser shots at an energy density, $E_{d}$, between 2.7 and $3.3 \mathrm{~J} / \mathrm{cm}^{2}$. The columns are $\sim 20 \mu \mathrm{m}$ long and $\sim 2 \sim 3 \mu \mathrm{m}$ in diameter. Moreover, surface-height profilometry performed using a Dektak II profiler revealed that most of the length of the microcolumns, $10-15$ $\mu \mathrm{m}$, protrudes above the original Si surface [14].

The microcolumn morphology changes if the atmosphere is changed during 


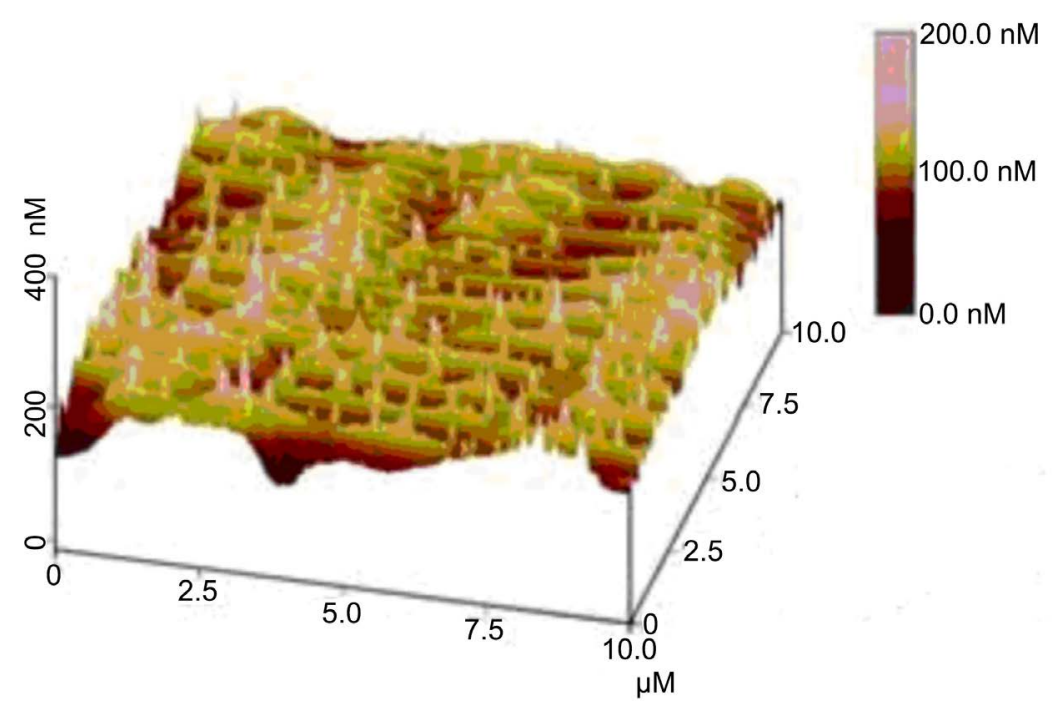

Figure 1. Three-dimensional AFM image of nanostructures after Nd:YAG laser irradiation with density of power $28 \mathrm{MW} / \mathrm{cm}^{2}$ on Ge surface [13].

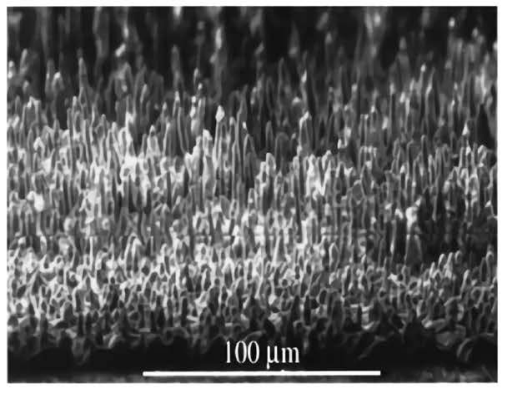

(a)

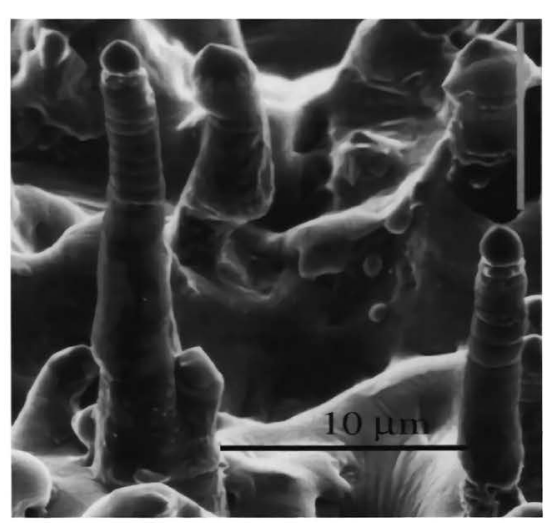

(b)

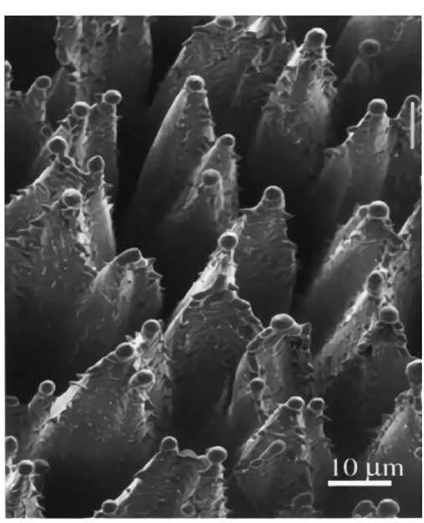

(c)

Figure 2. (a) SEM images of silicon microcolumns after 1000 laser shots in air at $3 \mathrm{~J} \cdot \mathrm{cm}^{-2}$ [14], (b) SEM image showing a change in Si microcolumn morphology controlled by the ambient gas composition at $E_{d} 2.7 \mathrm{~J} \cdot \mathrm{cm}^{-2}$. The arrows indicate the height achieved after 600 laser pulses in air $\left(\mathrm{N}_{2}-18 \% \mathrm{O}_{2}\right)$; the remainder of the columns was grown by 1200 laser pulses in $\mathrm{N}_{2}-5 \% \mathrm{O}_{2}$ [14]; (c) Walled Si structure produced by 2040 laser pulses at $E_{d}=1.5 \mathrm{~J} \cdot \mathrm{cm}^{-2}$ in $1 \mathrm{~atm}$ of SF 6 [15].

laser irradiation. Figure 2(b) shows several columns in a specimen that was first irradiated with 600 pulses in air and 1200 pulses in $\mathrm{N}_{2} / 5 \% \mathrm{O}_{2}$ [14].

The importance of the gas environment was emphasizing, when a plasma etchant, $\mathrm{SF}_{6}$, was using as the ambient gas during laser irradiation of silicon [14] [15]. In $\mathrm{SF}_{6}$ extremely long structures are produced that look at first like walls surrounding very deep central holes (see Figure 2(c)) [14] [15].

Laser-induced silicon nanostructures $\left(\lambda=0.8 \mu \mathrm{m}, \tau_{i}=100 \mathrm{fs}\right.$, number of pulses 200) with $d_{3}=90 \mathrm{~nm}$, which was generated after irradiation structures of changing polarization with $d_{2}=120 \mathrm{~nm}$, when orientation of vector $\boldsymbol{E}$ was changed on $90^{\circ}$ relatively to initial action. Power of laser irradiation was less in two time as for initial structure. Generated periodical structures [16] [17] are 
nanocolumns with height to $400 \mathrm{~nm}$ with spatial period $90 \mathrm{~nm}$ and orientation wave vector $\boldsymbol{g} \| \boldsymbol{E}$ ].

Where $\breve{g}$ is beam propagation direction.

An influence of pulses number on the processes of formation of laser-induced periodic nanostructures on titanium plate is observed in [18]. Sapphire laser system was used for the irradiation. It had next parameters: wavelength $800 \mathrm{~nm}$, repetition rate $1 \mathrm{kHz}$, pulse length $100 \mathrm{fs}$, beam diameter $4 \mathrm{~mm}$ and density of energy of irradiation $0.25 ; 0.75$ and $1.5 \mathrm{~J} / \mathrm{cm}^{2}$. Interference structures are generated for the irradiation with energy density $0.25 \mathrm{~J} \cdot \mathrm{cm}^{-2}$ [18]. Evolution of creation laser-induced surface structures is represented in Figure 3.

The period of the parallel periodic microstructures as a function of the number of laser pulses is shown in Figure 4 [18].

As shown in Figure 4, the period was gradually increased in the range of 10 70 pulses and increased in the range of $70-110$ pulses as the number of pulse increased.

Distance between the microdots along the hill of periodic microstructures as a function of the number of laser pulses is shown in Figure 5 [18]. As Figure 5 shows, the distance was increased in the range of 50 - 110 pulses. But these distances are correlated with height and width of interferogram bands (Figure 3).

Phenomenon of doubling of period of laser-induced surface structures is represented in Figure 6 [17] [18].

For the explanation of experimental data, which are represented in Figures 1-6, we must estimate all possible mechanisms of relaxation: kinetic and dynamical; and possible mechanisms of excitation: hierarchical photo ionization.
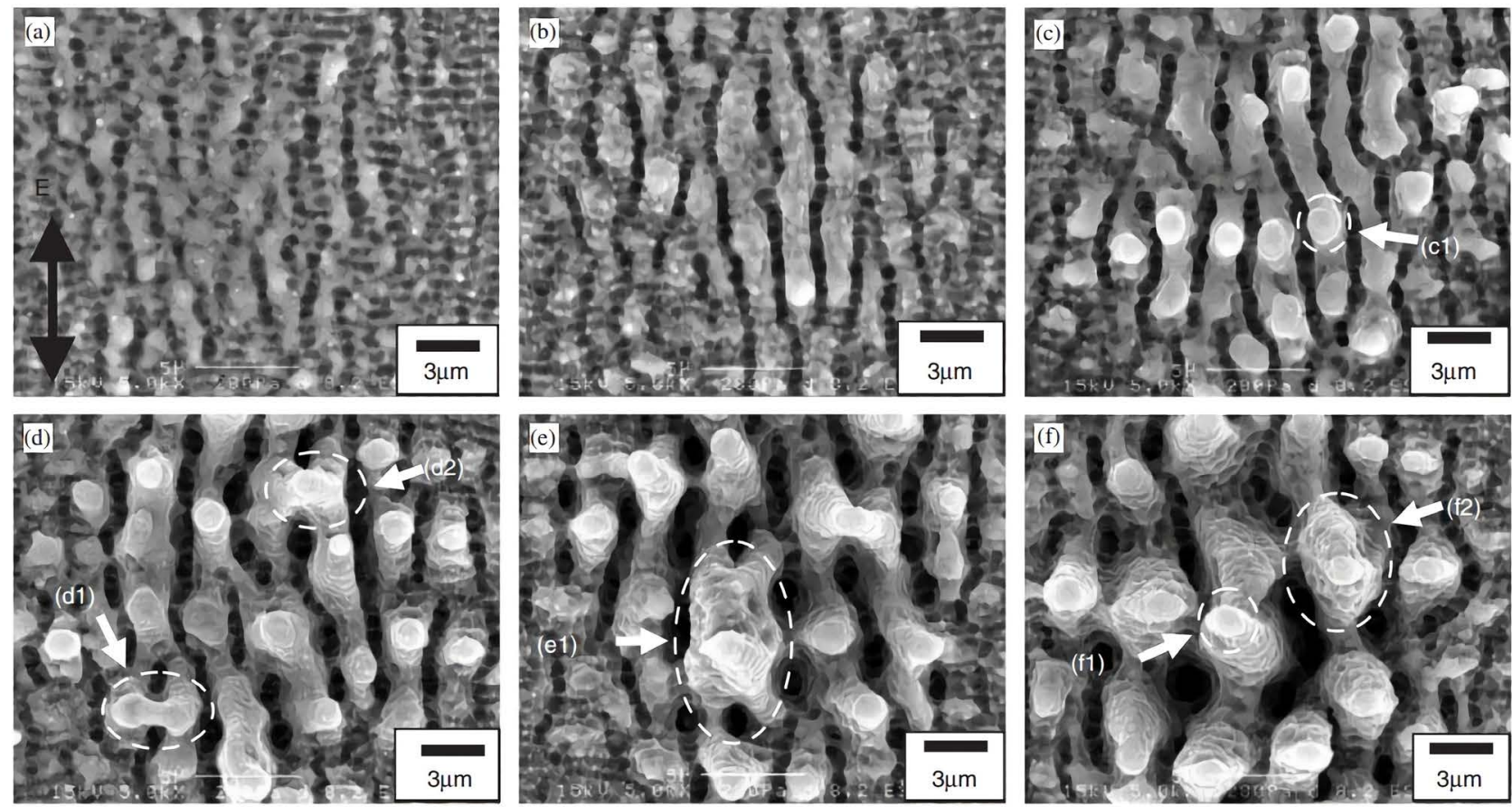

Figure 3. Microstructures produced on the titanium plate at the laser fluence of $0.75 \mathrm{~J} / \mathrm{cm}^{2}$ for (a) 10 , (b) 25 , (c) 50 , (d) 70 , (e) 90 and (f) 110 pulses [18]. 


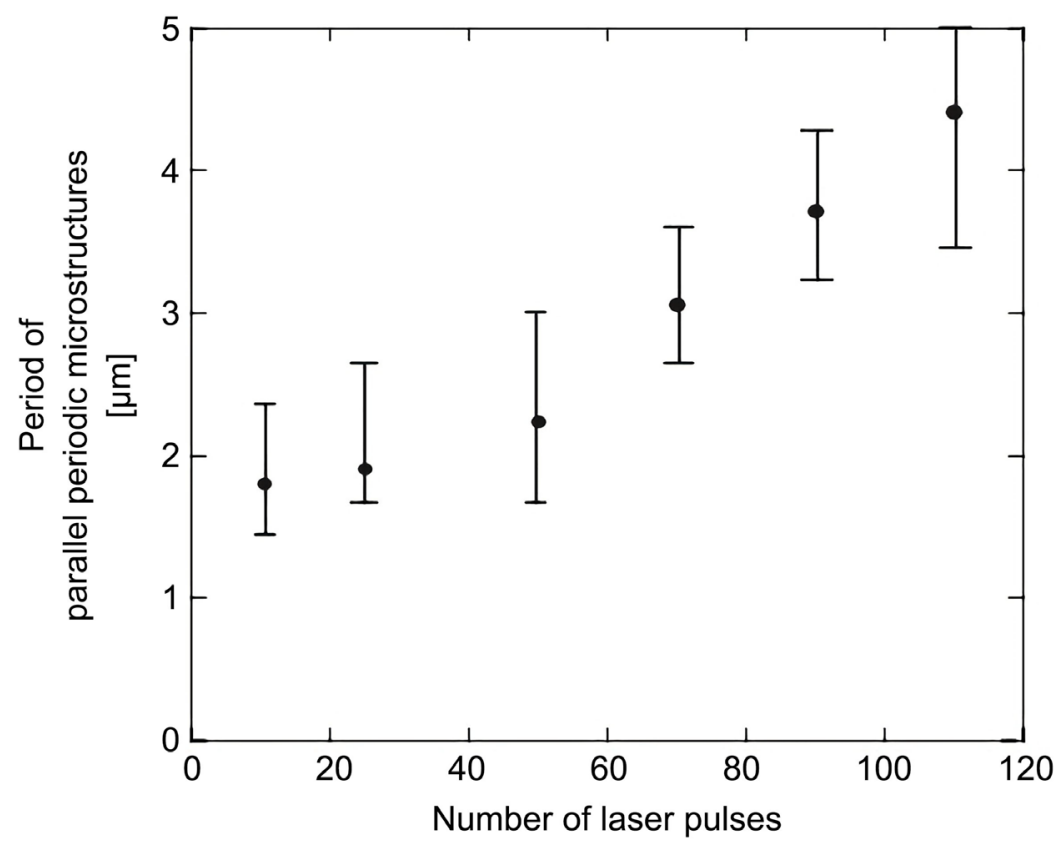

Figure 4. Period of parallel periodic microstructures as a function of number of laser pulses [18].

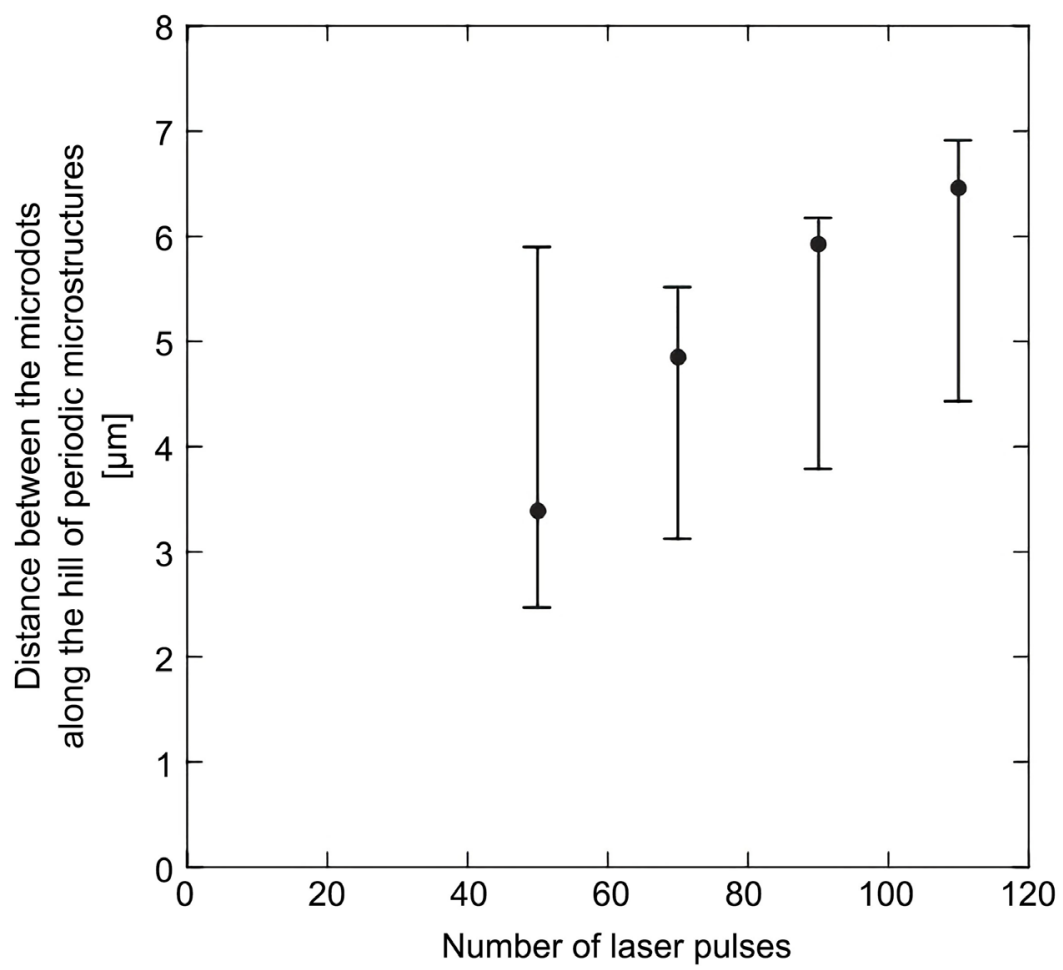

Figure 5. Distance between the microdots along the hill of periodic microstructures as a function of the number of laser pulses [18].

In this case we must include respective chain of relaxation times [1]. It is necessity for the more full representation and modeling real and possible physical processes for the respective regimes of interaction. 


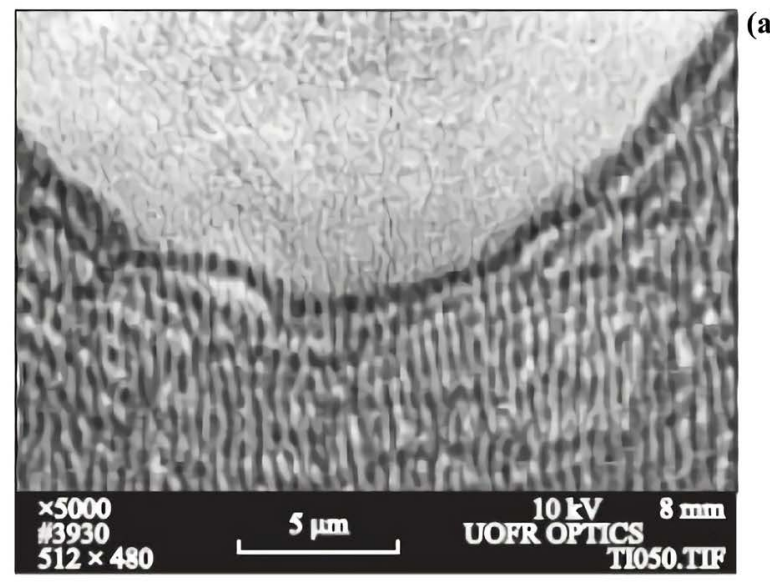

(a)

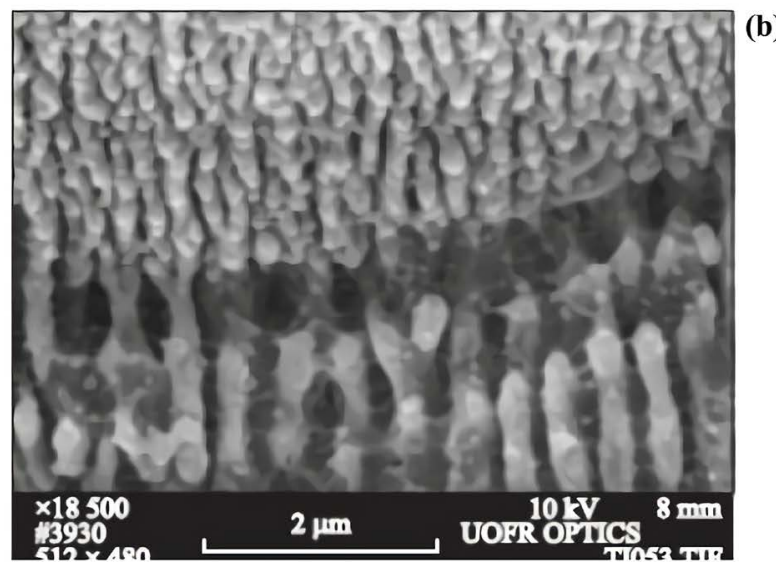

Figure 6. Relief of Ti surface after pulse linear polarized laser irradiation (power density $1.1 \mathrm{GW} / \mathrm{cm}^{2}$, number of pulses, 100): period of structures, $600 \mathrm{~nm}$, (b) region of transition from structures with period $600 \mathrm{~nm}$ to period $300 \mathrm{~nm}$ [17] [18].

Thus this representation of the modeling dangling bonds, which are created with help laser irradiation, is very effective method. It allows including in consideration effects of equilibrium, nonequilibrium and irreversible relaxations [1] [9].

Now we show the using of cascade model for the explanation experimental data of laser-induced phase transformations in silicon, germanium, carbon and titanium. It was called as case of the structural phases.

The question about the influence of saturation of excitation on effects of RO may be represented as process of transitions between stable and metastable phases too. Now we'll estimate the influence of parameters of irradiation (including spectral) on irreversible changes and transformations in $\mathrm{Si}$ and $\mathrm{Ge}$. Spectral dependences of absorbance of various structural modification of $\mathrm{Si}$ are represented in [1] [9]. Now we'll be estimated intensities of eximer, Ruby and Neodymium laser irradiation (wavelengths of irradiation are $0.248 \mu \mathrm{m}, 0.69 \mu \mathrm{m}$ and $1.06 \mu \mathrm{m}$ properly of silicon and germanium, which are necessary for the creation of proper irreversible changes in irradiated semiconductor. As shown in [1] [9], absorbance of the Neodimium laser radiation in silicon is equaled 60 - 
$100 \mathrm{~cm}^{-1}$, second harmonic of Neodimium laser, $10^{4} \mathrm{~cm}^{-1}$, eximer laser, $10^{6} \mathrm{~cm}^{-1}$. Crystal semiconductors $\mathrm{Si}$ and Ge have, basically, the structure of diamond. Volume atomic density of elementary lattices may estimate according to formula [1] [9]

$$
N_{a}=\frac{\rho N_{A}}{A},
$$

where $\rho$ : density of semiconductor, $N_{A}$ : Avogadro number, $A$ : a weight of one gram-atom. For Si $N_{a \mathrm{Si}}=5 \times 10^{22} \mathrm{~cm}^{-3}$ and for Ge $N_{a \mathrm{Ge}}=4.4 \times 10^{22} \mathrm{~cm}^{-3}$.

But Si and Ge may be crystallized in lattices of hexagonal, cubic, trigonal and monoclinic symmetry. Phase diagram of $\mathrm{Si}$ as function of coordination number is represented on Figure 7 [19].

Coordination number $(\mathrm{CN}) 8$ is corresponded to diamond lattice, CN 6: hexagonal lattice, $(\mathrm{CN}) 4$ and $(\mathrm{CN}) 3$ : other two lattices. It should be noted that melting temperatures of these phases are various. Volume density of $\mathrm{CN}$ is equaled CN. $N_{a}$. For diamond symmetry of lattice this value is $8 N_{a}$ [19].

Roughly speaking, transition from one phase to another for regime of saturation of excitation may be modeled as one-time breakage of proper numbers of chemical bonds, which are corresponded to the difference of $\mathrm{CN}$ of proper phases. For example, two bonds breakage is caused the phase transition from diamond to hexagonal structure. One bond breakage in the regime of saturation is caused to generation of laser radiation.

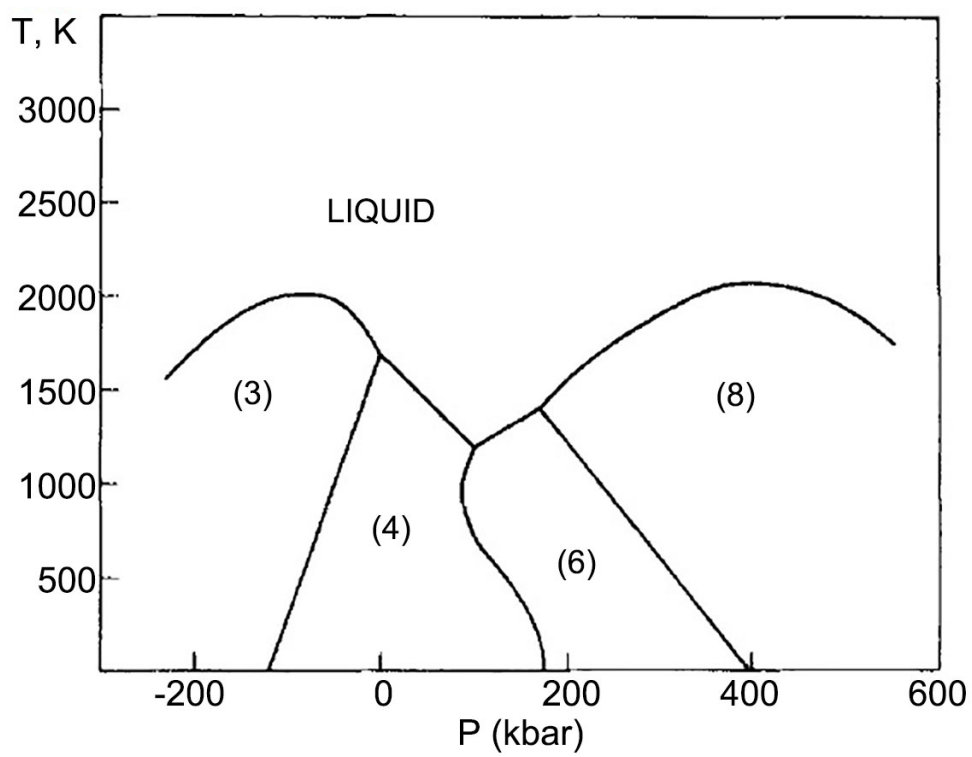

Figure 7. A schematic phase diagram for $\mathrm{Si}(\mathrm{CN})$. The coordination numbers $(\mathrm{CN})$ of the various phases are indicated. The diagram is based on common features of the phase diagrams of column IV elements as described by the references cited in Pistorius's review (8 in [15]). Starting from a high temperature $>3 \times 10^{3} \mathrm{~K}$ and subject to a constraint of average density $\langle\rho\rangle=\rho(4)$, a hot micronucleus will tend to bifurcate into the most stable phases (highest $T_{m}$ ) which straddle $\mathrm{Si}(4)$ in density. These are $\mathrm{Si}(3)$ and $\mathrm{Si}(8)$, as indicated by the diagram [19]. 
Results of calculation of volume densities of energy, which are necessary for breakage of proper number of bonds in regime of saturation of excitation, are represented in Table 1 [1] [8] [9].

It conceded that energies of all chemical bonds for elementary lattice are equivalent ( $\mathrm{Si}$ and Ge are pure homeopolar semiconductors) [1] [9]. For silicon energy of covalent bonds $\mathrm{Si}-\mathrm{Si}$ is equaled $1.2-1.8 \mathrm{eV}$; for germanium energy of covalent bonds $\mathrm{Ge}-\mathrm{Ge}$ are equaled $0.9-1.6 \mathrm{eV}$. Minimal values of these energies are corresponded to Pauling estimations. These values are corresponded the energy on one $\mathrm{CN}$ : according to radiation physics of status solid Zeits energy of creation one radiation defect in silicon is equal $12.7 \mathrm{eV}$ for diamond lattice [1] [9] [20].

Mechanisms of creation other laser-induced nanostructures may be explained on the basis cascade model of step-by-step excitation of corresponding type and number of chemical bonds in the regime of saturation of excitation [1] [8] [9]. According to this model decrease of symmetry of irradiated matter is occurred with increase of intensity of irradiation ( $\mathrm{Nd}$ laser irradiation of silicon, germanium and carbon) [1] [9].

But in [13] the explanation of creation laser-induced hexagonal phase on germanium surface is based on the Thomson-Benard phenomenon: generation of hexagonal phases in heated liquid on roaster. In our case heating of irradiated matter is generated in opposite direction from surface to deep and we have volume, but not surface as in Thomson-Benard effect [21] [22] [23], structures. This effect is observed for few liquids. Rayleygh-Chandrasekar theory is described this process as thermal-diffusive processes [22] [23]. In this case we have transition from more low volume symmetry to more high surface symmetry. Chandrasekar created the hydromagnetic theory of creation sunspots [23]. Thomson-Benard effect may be observed in other regimes of irradiation as in Figure 1. For laser-induced creation volume hexagonal structures on $G e$ we have inverse transition: from high volume symmetry (diamond modification) to more volume low symmetry (hexagonal symmetry) [21] [22] [23].

More intensive generation structures of Figure 2 may be explained with more intensive light absorption. Roughly speaking the number of disrupting chemical bonds (coordination numbers) $n_{d b}$ after absorption one photon may be determined with help next formula [1] [9] [20]

$$
n_{d b}=2 \ln \frac{h v}{E_{a}},
$$

Table 1. Volume density of energy $I_{v i}\left(10^{3} \mathrm{~J} / \mathrm{cm}^{3}\right)$, which is necessary for the breakage of proper number of chemical bonds in the regime of saturation of excitation in Si and Ge.

\begin{tabular}{ccccc}
\hline & $I_{V 1}$ & $I_{V 2}$ & $I_{v 4}$ & $I_{v 5}$ \\
\hline $\mathrm{Si}$ & $12.8-14.4$ & $25.6-28.8$ & $51.2-57.6$ & $63-72$ \\
$\mathrm{Ge}$ & $6.3-8.4$ & $12.6-16.8$ & $25.2-33.6$ & $31.5-42$ \\
\hline
\end{tabular}


where $h v$ : photon energy, $E_{a}$ : the energy of disruption the proper chemical bond (coordination number. For case of irradiation the silicon by pulses of eximer laser we have $h v=5 \mathrm{eV}, E_{a}=1.6 \mathrm{eV}$, and $n_{d b}=3.2$. This estimation shows the influence the spectra of irradiation on formation laser-induced structures [1] [8] [9] [20].

Formula (2) is true for $h v>E_{a}$ only. For case with irradiation silicon by eximer laser with wavelength $248 \mathrm{~nm} n$ is equaled from 2 to 3 .

More pure ionizing results were received for the irradiation silicon by femtosecond laser pulses in multipulse regime [9]. One quantum of eximer laser radiation may be ionized 3.2 chemical bonds according to formula (2). Therefore multipulse regime of the irradiation is caused the generation silicon structures with hexagonal and trigonal symmetry. Density of energy $2.7 \mathrm{~J} \cdot \mathrm{cm}^{-2}$ (Figure 2(b) and $1.5 \mathrm{~J} \cdot \mathrm{cm}^{-2}$ (Figure 2(c)) certificated this hypothesis. First regime allows receiving more thin and high structures with more low symmetry, which is stipulated more high intensity of irradiation. Second regime may be results of generation the structures with more low symmetry (no cubic). Atmosphere of irradiation must be having neglected influence on finishing picture. This conclusion is certificated by experimental data of Figure 2 [14] [15].

For light absorption on unstable centers (amorphous semiconductors) time characteristics haven't large observable influence on formation of irreversible changes in semiconductors. Here integral dose of irradiation has general meaning; therefore, in this case photochemical ionizing processes give basic contribution and processes of radiated relaxation are neglected.

With including of light reflection data of Table 1 must be increased on 20 - 30 percents for regimes of irradiation the Nedimium laser (both regimes) and 250 percents for eximer laser irradiation (reflectance of eximer laser radiation is equaled 60 percents) [1] [9].

In addition, we must remember that Ruby laser radiation for crystalline silicon has absorbance on order less as for amorphous, therefore for short regimes of irradiation the processes of bonds breakage may give more influence as for case of Nd-laser irradiation. Polycrystalline layer may include various crystalline phases. We can select condition of irradiation with creation on surface of silicon the nanostructures with various its fourth crystallographic modifications.

The role of irradiated atmosphere on formation of laser-induced surface nanostructures is shown in Figure 2(c). An oxidation processes are smooth out the surface (Figure 2(a) and Figure 2(b)). The decreasing of oxygen concentration is caused the more intensive growth of microcolumns (Figure 2(b) and nanocolumns [16].

The cascade model may be used for the explanation of experimental data of Figures 3-6. Titanium has two phases: hexagonal and body-centered cubic. But after heating the titanium to $882.5^{\circ} \mathrm{C}$ it has only cubic phase [9]. According to cascade model peaks of proper surface columns must have hexagonal symmetry, but its generation is attended with oxidation processes. Therefore the intensities 
of creation of the surface structures are various for two groups of pulses: for second group (70 - 110 pulses) is more intensive process as for first group of pulse (10 - 50 pulses). Among these regimes of irradiation may be received the doubling of period of interference pattern (Figure 6).

This method was used for the explanation of the experimental data about laser-induced transformations in various allotropic phases of carbon, included diamond, graphite, fullerenes and other [1] [8] [9], too.

\subsection{Volume Processes and Phenomena}

Problem of modelling volume processes of Relaxed Optics (RO) is connected with problem of increasing lifetime of basic elements of optoelectronic systems, including semiconductors, optical fibers and other.

In whole this problem [8] [9] [24] [25] [26] [27] is very complex problem. It connected with various nature of relaxation of first-order optical excitation. It may be simple two-three-stage, cascade, cyclic or more complex relaxation.

Therefore Relaxed Optical processes may be cause of nonlinear optical processes and conversely nonlinear optical processes may be caused of relaxed optical processes. Roughly speaking relaxed optical processes are the trace of interaction oh the light and matter in matter. Establishing the order of these processes is one of the most difficult problems of modeling mixed processes of relaxed optics. We must determine time, geometry and chain of proper processes.

Examples of mixing processes and phenomena of RO are pulse Ruby and Neodimium laser implantation of indium antimonite [1] [8] [9]; cascade processes of the creation of the laser-induced structures, including processes of laser sublimation and ablation [1] [9].

Basic NLO phenomena are connected with processes of intrinsic light scattering (self-absorption) on stable or metastable centers. So, the concentrations these centers in solid are equaled $\sim 10^{15}-10^{17} \mathrm{~cm}^{-3}$. Therefore we can use adiabatic approximation and basic formalisms of NLO are perturbation theory and nonlinear differential and integral equations.

Primary processes are caused of mechanisms of light scattering and local microscopic relaxation, secondary, macroscopic relaxation mechanisms. Therefore we must include these results for the explanation of real picture of interaction light and matter.

Observation of the volume laser-induced phase transformations processes (experimental data) was begin by Hersher [24] and first system researches were represented in B. Sharma $\mathrm{PhD}$ thesis [24].

Therefore methods for modeling of the laser-induced surface and volume phase transformations are various. This fact is caused of different conditions of radiation: self-absorption for surface and intrinsic absorption for volume. In first case we may have processes of one or two photon processes and for second case multiphoton processes. The problems of creation of the volume laser-induced phase transformations are another as creation the surface laser-induced phase 
transformations. This is represented on example of experimental data of volume laser-induced phase transformations in $4 \mathrm{H}-\mathrm{SiC}$ [9]. Influences of processes the multiphoton absorption and impact ionization are observed, too.

The problems of volume light scattering on metastable centers are analyzed for InSb and InAs magnetic sensors for nuclear reactors [9]. We show that using of $\mathrm{CO}_{2}$-laser irradiation is increased the lifetime of these sensors.

First experimental data of irreversible interaction the laser radiation and volume of solid were received by Hersher in 1964 [24].

He observed the surface interferograms after pulse Ruby-laser irradiation of germanium, silicon, indium antimonite a.o. [1] [9]. These results are beginning of researches of surface laser-induced phase transformations. The volume laser-induced phase transformations systematically were begun by B. S. Sharma [24]. He observed laser-induced damages in glasses after pulse Ruby laser irradiation.

Dependence of $E_{c r}^{2}$ from laser wavelength for various values of constant the development of avalanche is represented on Figure 8.

Dependence of threshold the N-pulse breakdown $I_{N}(N=200)$ in polymethylmethacrylate from concentration of various impurities $C_{i m}$ are represented in Figure 9 [25].

Dependences of threshold power of surface breakdown $P_{s b}$ from time of irradiation for two types Ruby samples [25] are represented in Figure 10.

Dependences of threshold intensity of laser-induced breakdown $I_{s b} \mathrm{NaCl}$ from temperature for various wavelengths of irradiation are represented in Figure 11 [25].

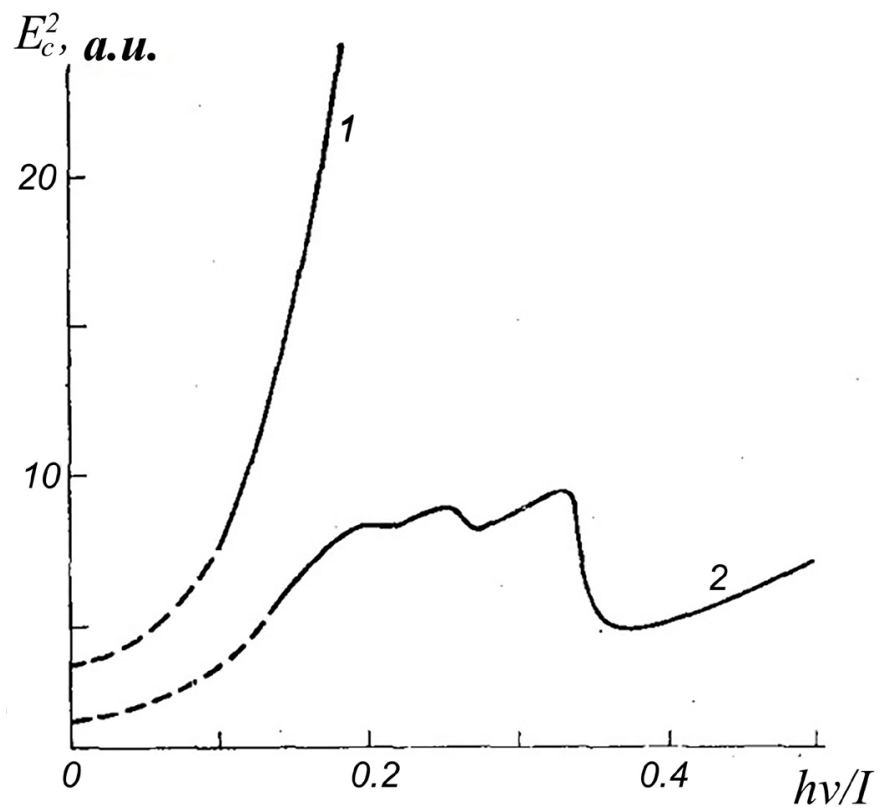

Figure 8. Dependence of $E_{c r}^{2}$ from laser wavelength for various values of constant the development of avalanche. $1-\gamma_{0}=3 \times 10^{-2}$ (picosecond pulses, $\tau_{i}=30 \mathrm{ps}$ ); $1-\gamma_{0}=10^{-5}$ (nanosecond pulses, $\tau_{i}=100 \mathrm{~ns}$ ) [25]. 


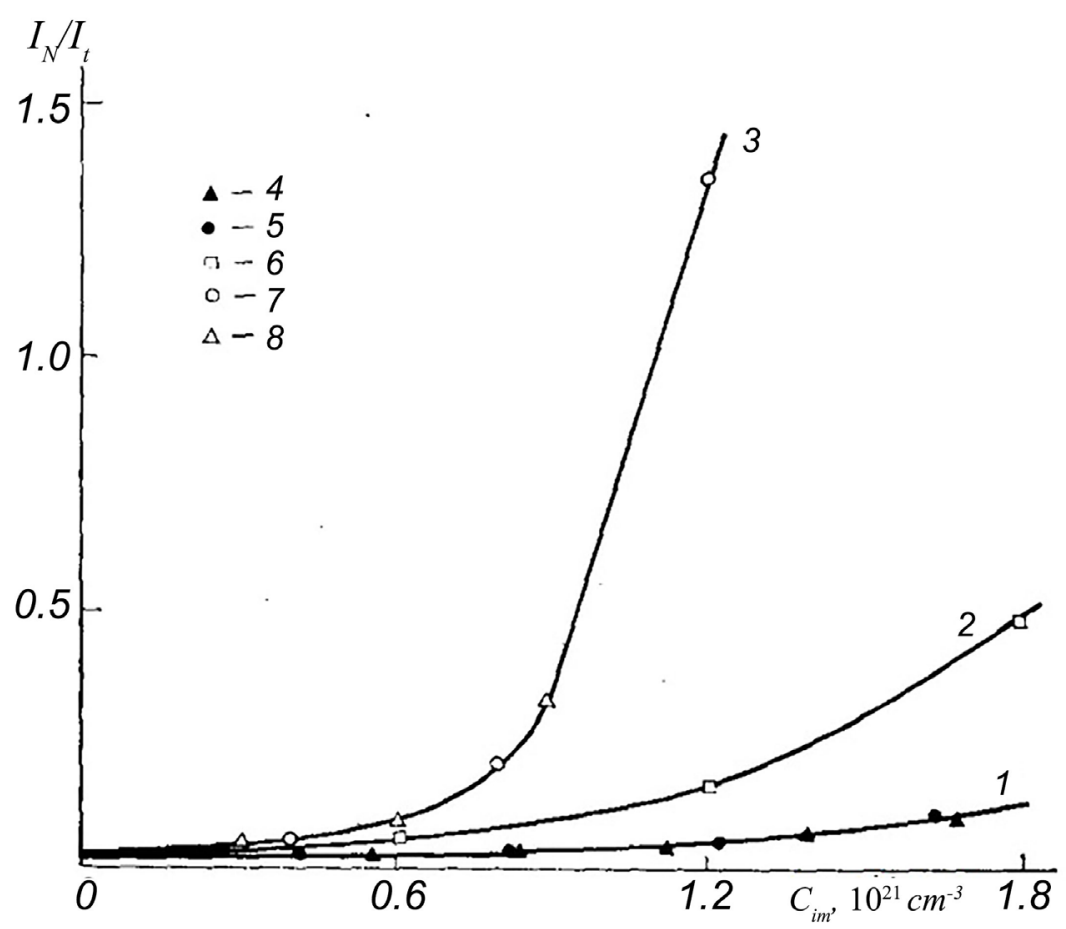

Figure 9. Dependence of threshold the $N$-pulse breakdown $I_{N}(N=200)$ in polymethylmethacrylate from concentration of various impurities $C_{i m}, 10^{21} \mathrm{~cm}^{-3}: 4$ : cyclohexanol; 5: butyronitrile; 6: ethanol; 7: butanol; 8: hexanol [25].

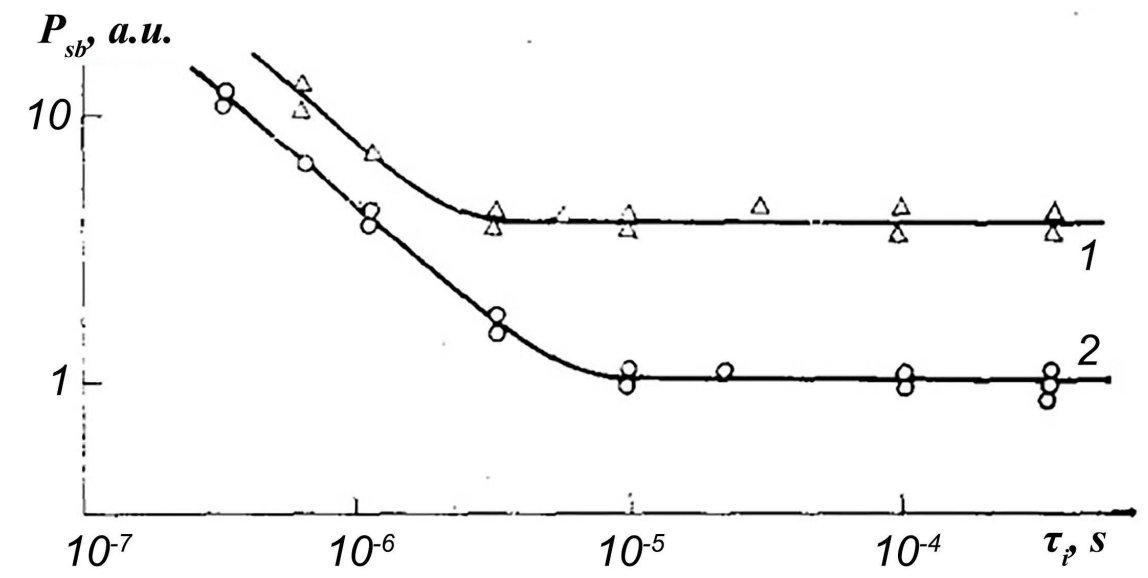

Figure 10. Dependences of threshold power of surface breakdown $P_{s b}$ from time of irradiation for two types Ruby samples [25].

Now we represent of some experimental data of RO, which will model. We select laser-induced surface phase transformations, which are caused the mechanisms of self-absorption; and volume phase transformations, which are connected with intrinsic mechanisms of light absorption. It allows observing basic peculiarities of proper physical processes and methods of its modeling.

The research of extensive measurements of damage thresholds for fused silica and calcium fluoride at 1053 and $526 \mathrm{~nm}$ for pulse durations $\tau$ ranging from 270 fs to $1 \mathrm{~ns}$ [26] is represented in Figure 12. 


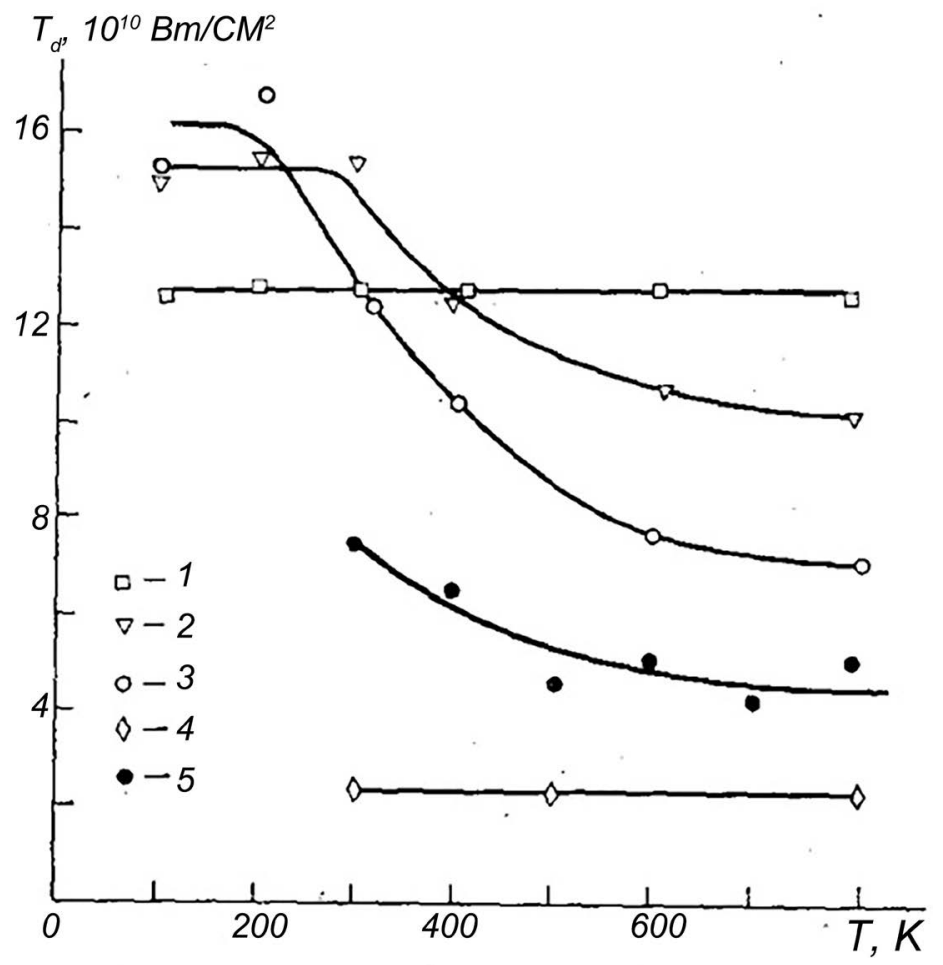

Figure 11. Dependences of threshold intensity of laser breakdown $I_{s b} \mathrm{NaCl}$ from temperature for various wavelengths of irradiation $\lambda, \mu \mathrm{m}$ : 0.53 (1); 0.69 (2); 1.06 (3); 10.6 (4); $2.76(5)[20]$

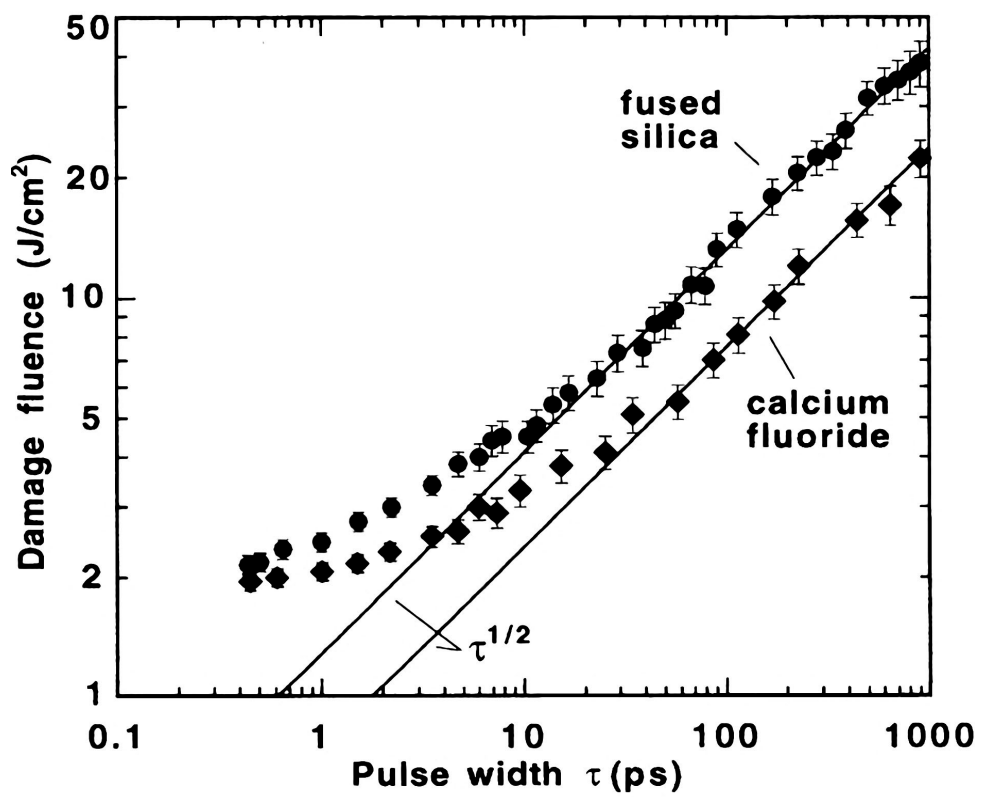

Figure 12. Observed values of damage threshold at $1053 \mathrm{~nm}$ for fused silica $(\bullet)$ and calcium fluoride $\left(\bullet\right.$. Solid lines are $\tau^{1 / 2}$ fits to long pulse results. Estimated uncertainty in the absolute fluence is $15 \%$ [26].

The explanation of experimental results of Figure 12, which is represented in [26], is next. In the long-pulse regime $(\tau>20 \mathrm{ps})$, the data fit well by a $\tau^{1 / 2}$ de- 
pendence, characteristic of the transfer of electron kinetic energy to the lattice and diffusion during the laser pulse. The damage is thermal in nature and characterized by the melting and boiling of the surface (Figure 13(a)). The damage occurs over the entire area irradiated. For long pulses, heating of the lattice and subsequent thermal damage can occur without significant collision ionization. For pulses shorter than $20 \mathrm{ps}$, the damage fluence no longer follows $\tau^{1 / 2}$ dependence and exhibits morphology dramatically different from that observed with long pulses. The damage appears as a shallow fractured and pitted crater characteristic of thin layers of material removed by ablation (Figure 13(b)).

Furthermore, short-pulse damage is confined to a small region at the peak of Gaussian irradiance distribution, where the intensity is sufficient to produce multiphoton ionization. With insufficient time for lattice coupling, there no is collateral damage. As a result, it can be many orders of magnitude smaller with short ( $\tau<10 \mathrm{ps}$ ) pulses than with long pulses. For the case of fused silica shown in Figure 14, the damages area produced by the $500 \mathrm{fs}$ pulse was two orders of magnitude smaller than that produced by the 900 ps pulse.

Pulse width dependence of threshold damage fluence for fused silica is represented in Figure 14 [26].

Measured and calculated (solid line) damage fluence for fused silica for 1053 and $526 \mathrm{~nm}$ are represented in Figure 15 [26]. The behavior of curves shows its correlation with absorption index. The more low damage fluence is corresponded to more large absorption index and shorter regime of irradiation is corresponded to faster regime of saturation.

Analogous pulse width dependence of threshold damage fluence was received for calcium fluoride (Figure 16 [26]).

Basic mechanisms of creation laser-induced damages in transparent materials are based on multiphotonic absorption of light. Few models and theories of these

(a)

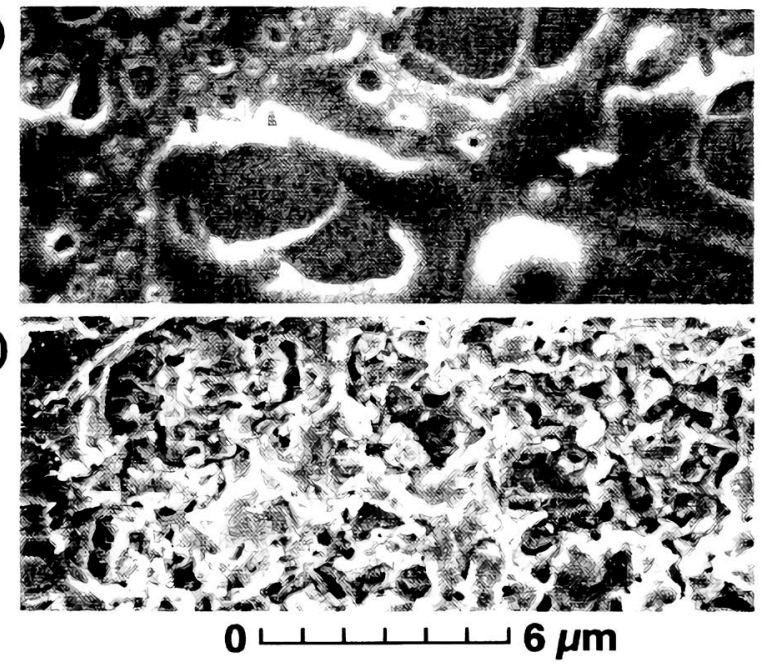

Figure 13. Scanning electron micrograth of front-surface damage of fused silica produced by $1053 \mathrm{~nm}$ pulses of duration (a) 900 ps, showing melting, and (b) $500 \mathrm{fs}$, showing ablation and fracture [26]. 


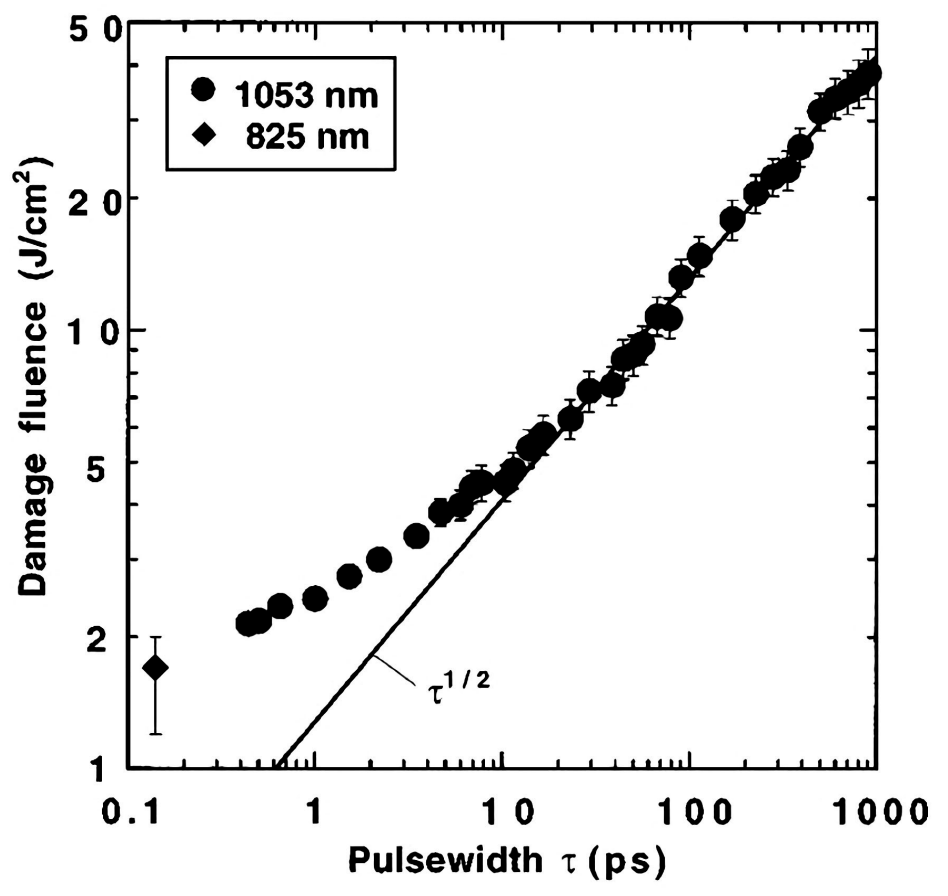

Figure 14. Pulse width dependence of threshold damage fluence for fused silica [26].

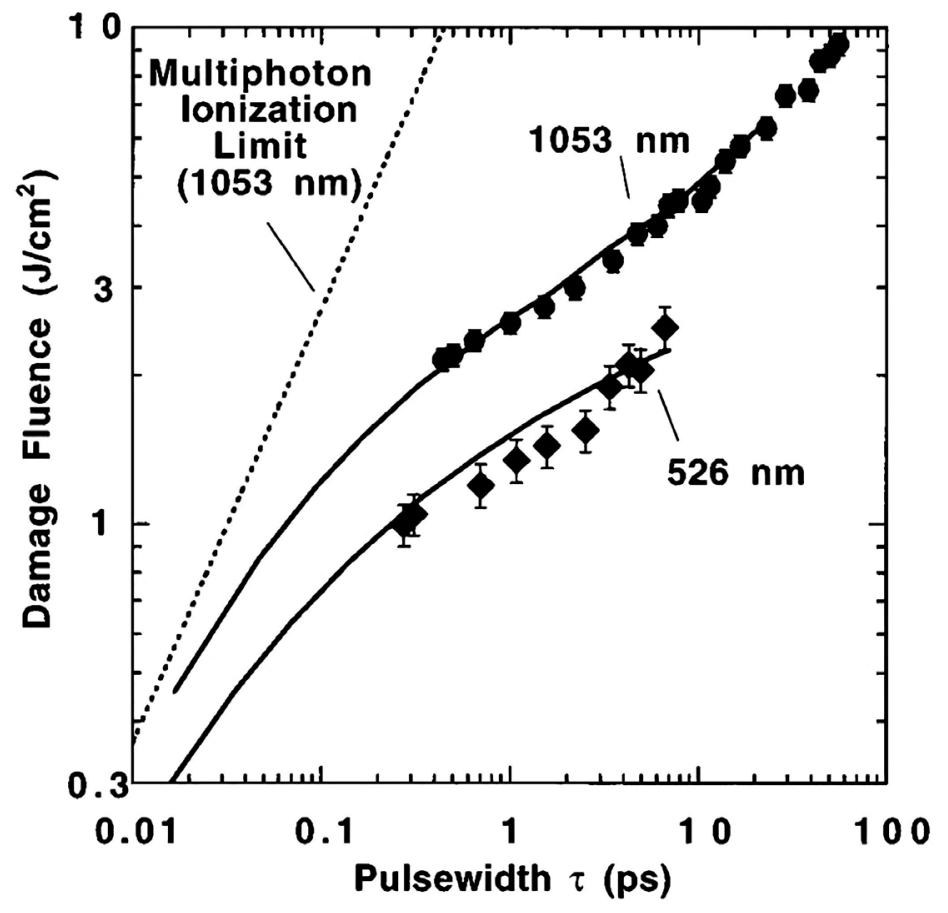

Figure 15. Measured and calculated (solid line) damage fluence for fused silica for 1053 and $526 \mathrm{~nm}$. Dashed line indicates calculated damage limit, due multiphoton ionization alone [26].

processes are represented. First system research of this problem was represented in B. S. Sharma Ph. D. thesis [24]. Therefore we use his work as basis in the explanation of respective experimental data. 


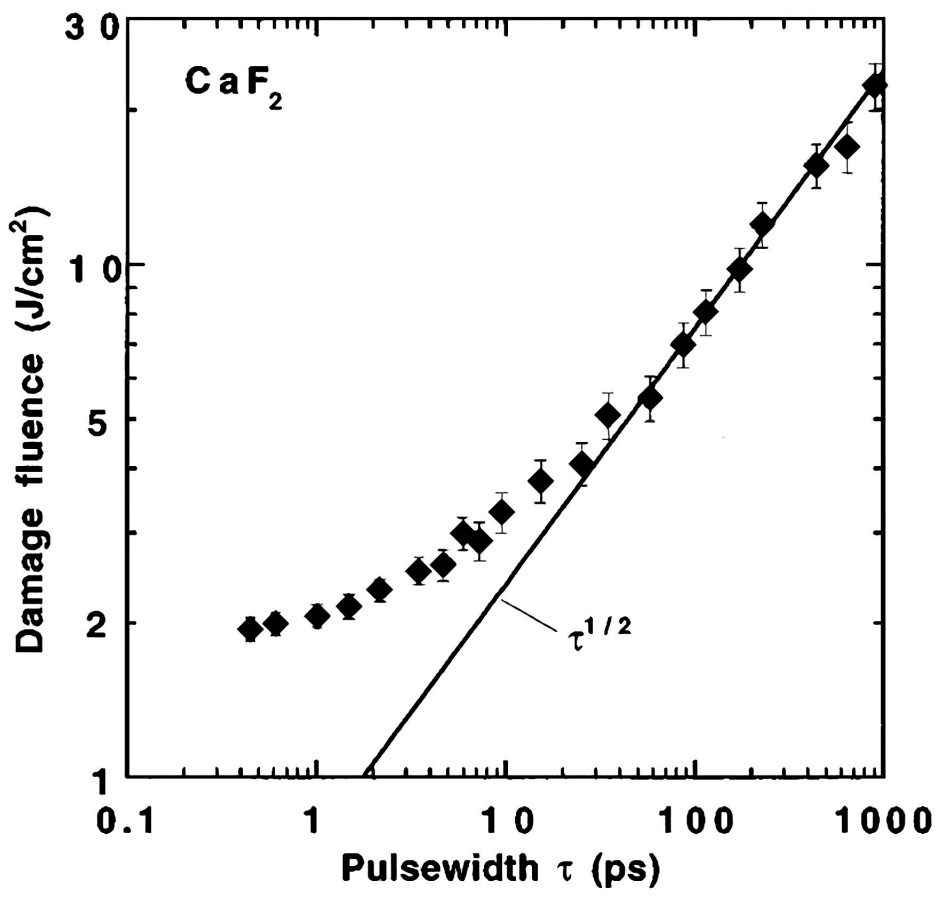

Figure 16. Pulse width dependence of threshold damage fluence for calcium fluoride [26].

Basic mechanical mechanisms of laser-induced breakdown according by B.S. Sharma [24].

The three basic breakdown mechanism of Ruby-laser breakdown in glasses are:

1) electrostriction;

2) acoustic wave generation by stimulated Brillouin scattering;

3) multiphoton ionization and plazma formation.

1) Electrostriction [24]

Stresses caused by the direct action of the light on dielectrics are due to:

a) Direct light pressure. This phenomenon is consistent with the concept of the momentum of electromagnetic waves. If electromagnetic waves falls on an absorbing material and isn't reflected, then its momentum is transmitted to the material in accordance with the conservation law of linear momentum. The rate of transfer of this momentum will be the force experienced by the body and is termed the light pressure. This rate of transfer in the case of totally absorbing body will be the momentum density of the radiation, which is equal to the energy density divided by speed of light. Thus the maximum light pressure will be

$$
p=\frac{I}{c} \text {. }
$$

For the energy flux $I=5 \times 10^{9} \mathrm{~W} \cdot \mathrm{cm}^{-2}$ this pressure is equaled $p=1.7 \times 10^{5}$ $\mathrm{N} \cdot \mathrm{m}^{-2}$. For picosecond laser: $I=5 \times 10^{12} \mathrm{~W} \cdot \mathrm{cm}^{-2}, p=1.7 \times 10^{8} \mathrm{~N} \cdot \mathrm{m}^{-2}$; and for $I=$ $5 \times 10^{15} \mathrm{~W} \cdot \mathrm{cm}^{-2}, p=1.7 \times 10^{11} \mathrm{~N} \cdot \mathrm{m}^{-2}$. For Ruby-laser is very small value.

b) Electrostriction pressure. In this case deformation produced by electric 
stresses. Deformation of a dielectric in an electric field is determined partly by the Maxwell stresses and partly by a dependence of a dielectric constant upon the strain. In a electric field, a dielectric tends to assume a configuration such to reduce the total energy to the minimum. If, as is usually for glasses, the dielectric constant increases as the density increases, then Maxwell stresses and the varying dielectric constant conspire to make the density increased then the field is applied. In some substances the dielectric constant decreases with increasing density. In such a case the state of minimum energy may be accompanied by a decrease in density.

These deformations and the resulting electrostriction pressure are proportional to the square of electric field and are independent of its direction. The electrostriction pressure can be evaluated by equating the work done on the dielectric to compress it to the change in the electrical energy.

Consider a condenser of volume $V$ immersed in a compressible fluid. The changes of electrical energy when the fluid contracts by an amount $\Delta V$ are

$$
\frac{E^{2}}{4 \pi} V\left(\frac{\mathrm{d} \varepsilon}{\mathrm{d} V}\right) \Delta V
$$

where $\mathrm{d} \varepsilon$ is the change in the dielectric constant due to compression.

Equating this energy to the amount of work done on the fluid $p_{e l} \Delta V$

$$
p_{e l} \Delta V=\frac{E^{2}}{4 \pi} \rho V\left(\frac{\mathrm{d} \varepsilon}{\mathrm{d} V}\right) \Delta V .
$$

Since

$$
\frac{\Delta \rho}{\rho}=-\frac{\Delta V}{V},
$$

where $\rho$ is the mass density, we get

$$
p_{e l}=\frac{E^{2}}{4 \pi} \rho\left(\frac{\mathrm{d} \varepsilon}{\mathrm{d} \rho}\right),
$$

where

$$
\gamma=\rho\left(\frac{\mathrm{d} \varepsilon}{\mathrm{d} \rho}\right)
$$

is a constant having a value from 0.6 to 1 for glasses.

Maximum electrostriction pressure for an incident beam of intensity $I=5 \times$ $10^{9} \mathrm{~W} \cdot \mathrm{cm}^{-2}$ will be (for $\gamma=1$ ) equal to:

$$
P_{e l}=1.6 \times 10^{6} \mathrm{~N} \cdot \mathrm{m}^{-2},
$$

which is again very small when compared with the strength of the glasses.

2) Stimulated Brillouin scattering [24]

In the normal Brillouin scattering (Figure 17) an incident light beam is scattered by thermally excited acoustic waves. Scattered light is shifted in wavelength and the energy difference is taken up by the acoustic wave. A consideration of the energy and the phase vector conservation leads to the relations 


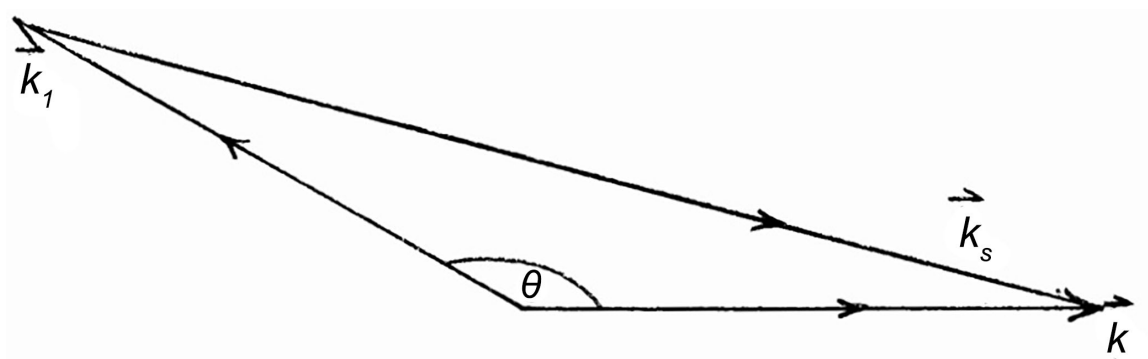

Figure 17. Rough schema of Brillouin scattering [24].

$$
\begin{gathered}
\omega=\omega_{1} \pm \omega_{s}, \\
\boldsymbol{k}=\boldsymbol{k}_{1} \pm \boldsymbol{k}_{s},
\end{gathered}
$$

where $\omega, \boldsymbol{k}$ are the frequency and the phase vector of the incident light; $\omega_{1}, \boldsymbol{k}_{1}$ for the scattered light and $\omega_{s}, \boldsymbol{k}_{s}$ for the acoustic wave.

The well-known Brillouin expression for the frequency shirt is:

$$
\omega-\omega_{1}= \pm \omega_{s} \cong \frac{2 \vartheta_{s}}{c} n \omega \sin \frac{\theta}{2},
$$

where $\vartheta_{s}, c / n$ are the acoustic and optical phase velocities in the medium, $n$ is the index of refraction, which will be essentially the same for the incident and scattered light rays in isotropic media, and $\theta$ is their angular separation.

These relations imply that acoustic waves can either be generated or annihilated in the scattering process. When acoustic waves are generated, enhancement in light scattering can occur. With a weak exciting source enhancement is negligible but with a Q-switched laser pulse, the effect is profound. The process leads to a marked regenerative behavior i.e. amplification on the scattered light. A brief sketch of the dynamics of the stimulated Brillouin scattering following Chiao et al. [24] [25] is present below.

Coupling of the light waves with the sound waves is due to the electrostriction, the compression or expansion of the material by the electric fields of the light wave. Consider two beams of light at slightly different frequencies in the medium

$$
\boldsymbol{E}(\boldsymbol{r}, t)=\boldsymbol{E}_{0} \cos (\boldsymbol{k} \cdot \boldsymbol{r}-\omega t+\varphi)
$$

and

$$
\boldsymbol{E}(\boldsymbol{r}, t)=\boldsymbol{E}_{0} \cos \left(\boldsymbol{k} \cdot \boldsymbol{r}-\omega t+\varphi_{1}\right) .
$$

Let the sound wave be described by

$$
P(\boldsymbol{r}, t)=P_{0} \cos \left(\boldsymbol{k} \cdot \boldsymbol{r}-\omega_{s} t+\varphi_{s}\right) .
$$

The process of the electrostriction causes a local compression $\delta \mathrm{V}$, which in the presence of a local pressure $P$ due to the sound wave does work by an amount

$$
\delta W=P(\vec{r}, t) \delta V(\vec{r}, t) .
$$

The average power transferred to the sound wave per unit volume is: 


$$
\frac{\mathrm{d} u_{s}}{\mathrm{~d} t}=-\int \frac{\mathrm{d}^{3} \boldsymbol{r}}{V_{0}} \int_{-T / 2}^{T / 2} \frac{\mathrm{d} t}{T} P(\boldsymbol{r}, t) \frac{\partial}{\partial t} V(\boldsymbol{r}, t),
$$

where $u_{s}$ is the energy density of the sound wave,

$$
\frac{\delta V(\boldsymbol{r}, t)}{V_{0}}=\frac{1}{B} \delta P_{e l}(\boldsymbol{r}, t)=\frac{\gamma}{8 \pi B} \delta\left[2 \boldsymbol{E}(\boldsymbol{r}, t) \cdot \boldsymbol{E}_{1}(\boldsymbol{r}, t)\right],
$$

where $B$ is the bulk modulus of material. Considering only the resonant term equation (17) can be written as

$$
\frac{\mathrm{d} u_{s}}{\mathrm{~d} t}=\frac{\gamma}{16 \pi B} P_{0}\left(\boldsymbol{E}_{0}, \boldsymbol{E}_{1}\right) \omega_{s},
$$

where we have used $\boldsymbol{k}=\boldsymbol{k}_{1}+\boldsymbol{k}_{s}, \omega=\omega_{1}+\omega_{s}$ and $\varphi=\frac{\pi}{2}+\varphi_{1}+\varphi_{s}$.

The polarization produced by the sound wave gives rise to a dipole moment $\delta \boldsymbol{\mu}$, which in the presence of the local electric field $\boldsymbol{E}_{1}$, leads to an energy transfer of:

$$
\delta W=\boldsymbol{E}_{1}(\boldsymbol{r}, t) \cdot \delta \boldsymbol{\mu}(\boldsymbol{r}, t) .
$$

Thus the average power transferred to the scattered wave at frequency $\omega_{\perp}$ is

$$
\frac{\mathrm{d} u_{1}}{\mathrm{~d} t}=\int \frac{\mathrm{d}^{3} \boldsymbol{r}}{V_{0}} \int_{-T / 2}^{T / 2} \frac{\mathrm{d} t}{T} \boldsymbol{E}(\boldsymbol{r}, t) \frac{\partial \boldsymbol{P}(\boldsymbol{r}, t)}{\partial t},
$$

where $\boldsymbol{P}(\boldsymbol{r}, t)$, the induced polarization is given by

$$
\delta \boldsymbol{P}(\boldsymbol{r}, t)=\frac{\Delta \varepsilon}{4 \pi} \boldsymbol{E}(\boldsymbol{r}, t)=\frac{\gamma}{4 \pi B} \delta[\boldsymbol{P}(\boldsymbol{r}, t) \cdot \boldsymbol{E}(\boldsymbol{r}, t)] .
$$

Again considering the resonant terms only we get

$$
\frac{\mathrm{d} u_{1}}{\mathrm{~d} t}=\frac{\gamma}{4 \pi B} P_{0}\left(\boldsymbol{E} \cdot \boldsymbol{E}_{10}\right) \omega_{1} .
$$

If the plane of polarization of the $\boldsymbol{E}$ be perpendicular to the plane of the scattering of $\boldsymbol{E}_{1}$, we can write:

$$
\left(\frac{\mathrm{d} u_{s}}{\mathrm{~d} t}\right)_{\text {gain }}=\frac{\gamma \omega_{s}}{16 \pi B} P_{0} \boldsymbol{E}_{0} \boldsymbol{E}_{10}
$$

and

$$
\left(\frac{\mathrm{d} u_{1}}{\mathrm{~d} t}\right)_{\text {gain }}=\frac{\gamma \omega_{1}}{16 \pi B} P_{0} \boldsymbol{E}_{0} \boldsymbol{E}_{10}
$$

The energy density of the sound wave $u_{s}=\frac{P_{0}^{2}}{2 B}$ will decrease due to the losses according to

$$
\left(\frac{\mathrm{d} u_{s}}{\mathrm{~d} t}\right)_{\text {loss }}=-\frac{u_{s}}{\tau_{s}}
$$

Similarly the energy density of the scattered wave $u_{1}=\frac{\varepsilon_{1} E_{0}^{2}}{8 \pi} \quad$ will decrease as 


$$
\left(\frac{\mathrm{d} u_{1}}{\mathrm{~d} t}\right)_{\text {loss }}=-\frac{u_{1}}{\tau_{1}}
$$

For the build up of the sound wave and the scattered wave gain must exceed the losses that are

$$
\frac{\gamma \omega_{s}}{16 \pi B} P_{0} \boldsymbol{E}_{0} \boldsymbol{E}_{10} \geq \frac{1}{\tau_{s}} \frac{P_{0}^{2}}{2 B}
$$

and

$$
\frac{\gamma \omega_{1}}{16 \pi B} P_{0} \boldsymbol{E}_{0} \boldsymbol{E}_{10} \geq \frac{1}{\tau_{s}} \frac{\varepsilon_{1} E_{10}^{2}}{8 \pi} .
$$

Multiplying (28) and (29) we get the threshold condition for gain as

$$
\frac{E_{0}^{2}}{8 \pi} \geq \frac{1}{\omega_{s} \tau_{s} \omega_{1} \tau_{1}} \frac{2 B \varepsilon_{1}}{\gamma^{2}} .
$$

Thus in order to have a catastrophic build up of sound and the Brillouin scattered wave, the incident intensity must be large enough to overcome the losses. For the case of fused quartz various constants are:

$$
\begin{gathered}
\gamma=0.6 ; \tau_{1}=3 \times 10^{-9} \mathrm{~s} ; \tau_{s}=5 \times 10^{-9} \mathrm{~s} ; B=10^{12} \text { dynes } \cdot \mathrm{cm}^{-2} ; \\
\omega_{1}=10^{15} \mathrm{~s}^{-1} ; \omega_{s}=10^{10} \mathrm{~s}^{-1} ; \frac{c E_{0}^{2}}{8 \pi} \geq 100 \varepsilon_{1} \mathrm{MW} \cdot \mathrm{cm}^{-2} .
\end{gathered}
$$

$\mathcal{E}_{1}$ is roughly 2.3 , so the threshold energy density for the stimulated Brillouin scattering is $230 \mathrm{MW} \cdot \mathrm{cm}^{-2}$. Kroll in 1965 [27] considered the problem of photoelectric instability in quartz and sapphire under illumination by an intense laser pulse. He solved the phenomenological equations, describing the photoelastic coupling between the electromagnetic and the elastic waves by a Lagrangian density

$$
L_{i n t}=-\frac{1}{8 \pi} p_{i j k l} D_{i} D_{j} \frac{\partial u_{k}}{\partial x_{l}},
$$

where $u_{k}$ refers to the displacements; $D_{i}$, the electric displacement vector and $p_{i j k l}$ is the Pockel's elasto-optic constant. Results of his calculation show that the growth of a photoelastic excitation is governed primarily by an exponential factor of the form $\left(1.25 W_{l}^{1 / 2} x^{1 / 2}-\vartheta_{s} \alpha t\right)$, where $W_{l}$ is the laser energy in $\mathrm{J} \cdot \mathrm{cm}^{-2}, x$ is the interaction length, $\mathcal{\vartheta}_{s}$ is the sound velocity, $\alpha$ the attenuation at sound wavelength, and $t$ is the laser pulse duration. The backward instability is well developed when this exponent is of the order of ten or more. Kroll gives the following relation for the power carried by the elastic wave in terms of the power carried by the Stokes shifted wave,

$$
P_{s}=\frac{\omega_{s}}{\omega} \frac{\vartheta_{s} t}{x} P_{1}
$$

where $\omega_{s}$ is the elastic wave frequency, $\omega$ the incident laser frequency, $P_{s}$ is the acoustic power and $P_{1}$ the power in the Stokes shifted wave. The build up of $P_{1}$, is limited by the failure of the linear approximation and in particular by the 
reaction back upon the incident laser power. Budin et al. [9] measured the Stokes light power for various incident laser energies for a silicate glass. From their data, for a focused Ruby laser energy of $0.07 \mathrm{~J}$ or $210 \mathrm{~J} / \mathrm{cm}^{2}$ the Stokes shifted is $P_{1}=2000 \mathrm{~W}$. Using Kroll's criterion for the instability [9] [27] we can calculate $x$.

$$
1.25 W_{l}^{1 / 2} x^{1 / 2}-\vartheta_{s} \alpha t=10
$$

Substituting all the values we get $x=0.3 \mathrm{~cm}$ or the $P_{s}=2 \times 10^{-4} \mathrm{~W}$. These yield an acoustic wave intensity of approximately $6 \mathrm{~W} / \mathrm{cm}^{2}$. Even if we assume that all this acoustic power is absorbed in the focal volume, the maximum value of the effective absorption due to this mechanism is $\xi=0.1$, which is very much smaller than the minimum effective absorption needed for mechanical breakdown as deduced from energy considerations.

\subsection{Laser-Induced Optical Breakdown}

Problems of the observation the laser-induced optical breakdown and shock processes in matter as Nonlinear Optical (NLO) and Relaxed Optical (RO) processes are connected with acoustic (thermal) and electromagnetic (plasma and Nonlinear optical) nature [2] [7] [8] [9] [28]-[33]. These processes may be connected with diffractive stratification of laser beam, self-focusing, self-trapping, generation of supercontinuum radiation (ordered: Cherenkov radiation, and disorder: plasma radiation) [7] [8] [34] [35] [36].

We present this problem from one point of view for all media from gases to solid [2] [3] [7] [8] [28]-[33]. Unfortunately, the first attempt of observation this problem in main detail in whole are represented in [7] [8] only.

According to [2] [4] [7] [8], optical breakdown is understood as catastrophic damage caused by strong laser radiation. The cause of optical breakdown is avalanche ionization [2] [4] [7] [8]. This process is differed from heat breakdown, which is result of laser-induced heat of irradiated matter, to direct optical multiphoton ionization. Roughly speaking the optical breakdown is result of rapid introducing energy to matter with laser help. Optical breakdown determines a limit laser intensity of laser radiation, which irradiated matter can absorb.

In whole this problem [2] [4] [7] [8], is very complex problem. From physical-chemical point of view the optical breakdown is the regime of fool breakage of all chemical bonds in irradiated matter in zone of laser irradiation [7] [8]. In this case we can determine the threshold of breakdown of irradiated matters with help methods of RO (cascade model of excitation the proper chemical bonds in the regime of saturation the excitation) [6] [7] [8] [9]. This regime may be received with help three ways. First is thermal. In this case the basic relaxation of first order processes of optical excitation is thermal [2] [4]. As example of this process may be continuous laser irradiation of matter in self-absorption range of absorption spectrum [2] [4]. Second is plasma. In this case the main role of the optical breakdown has process of formation "collective" electromagnetic (electron-ionic) process [2] [4]. The examples of this process are 
the irradiation in the millisecond or nanosecond regimes of irradiation [2] [3]. In this case laser-induced plasma radiated continuum optical spectra in all direction (star effect) [2]. Third is direct optical [7] [8]. In this case we have direct multiphoton ionization and these processes have oriental nature [4] [7] [8]. The second order irradiation has Cherenkov nature [7] [8] [34]. The experimental data were received for picosecond and femtosecond regimes of irradiation [7] [8] [28]-[33]. This differentiation is connected with various nature of relaxation of first-order optical excitation. Thus we have three ways for the receiving of laser-induced breakdown.

The process of laser-induced optical breakdown is closely related to the phenomena of self-focusing and the appearance of laser-induced filaments [9] [37].

Firstly, theory of these processes (types 1 and 2) was made for the gases [2] [4] [27]. Later this theory was adapted to solid [2] [4] [24]. Theories of third type processes were created and developed in [7] [8].

Basic ways of possible applications of laser-induced optical breakdown processes for the creation of new multifunctional elements of modern optoelectronics and new laser technologies are discussed, too.

Here are the experimental results that show the cascade nature of these processes.

Refractive index map for two times, which was measured in [28], is represented on Figure 18. This picture shows the process of the development optical breakdown in water.

More complex experimental data, which are included optical breakdown, were received in [29] [30] (Figure 19).

Sectional area of receiving structures was $\sim 22 \mu \mathrm{m}$, the depth of $\sim 50 \mu \mathrm{m}$. As seen from Figure 19(c) we have five stages disordered regions, which are located at a distance from 2 to $4 \mu \mathrm{m}$ apart vertically [29] [30]. Branches themselves in this case have a thickness from 150 to $300 \mathrm{~nm}$. In this case there are lines in the irradiated nanocavity spherical diameter of from $10 \mathrm{~nm}$ to $20 \mathrm{~nm}$. In this case irradiated structures have crystallographic symmetry of the initial structure.

(a)

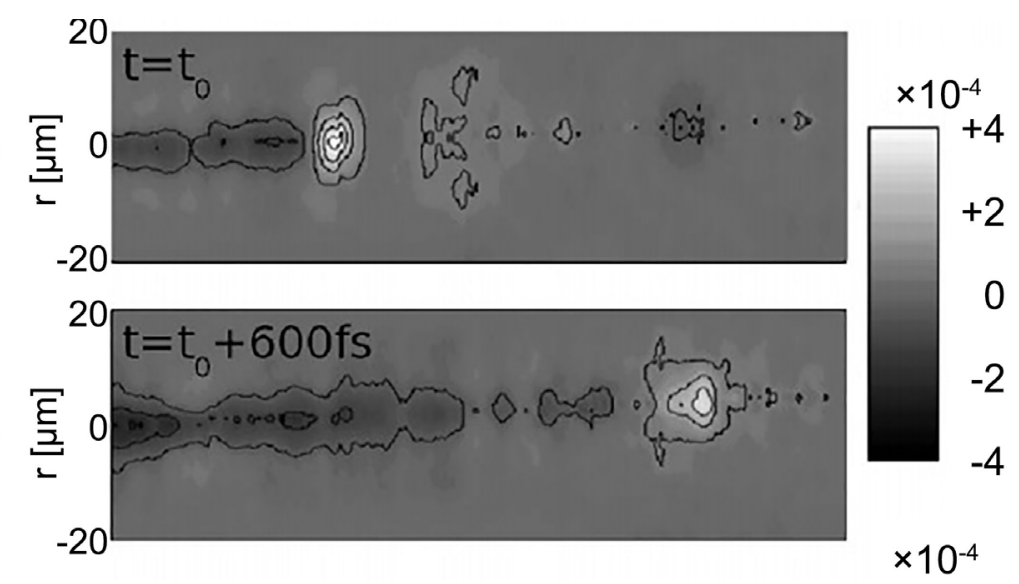

Figure 18. (a) and (b) Refractive index map of the filament taken at two different times and showing the formation of the plasma channel. Horizontal scale: propagation axis ranging from $9.41 \mathrm{~mm}$ (left) to $9.68 \mathrm{~mm}$ (right) from the input window [28]. 
(a)

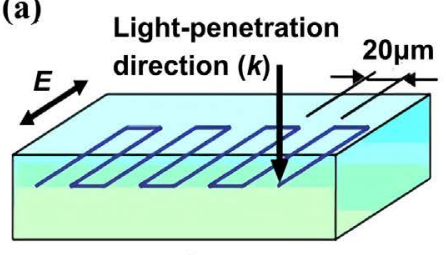

(b)

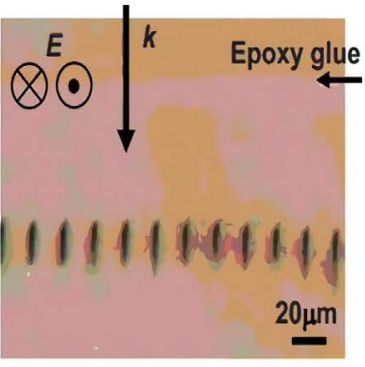

(c)

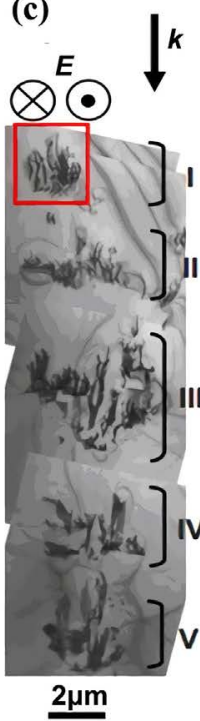

(d)

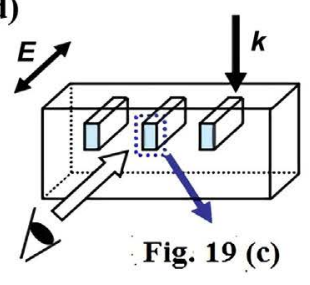

(e)

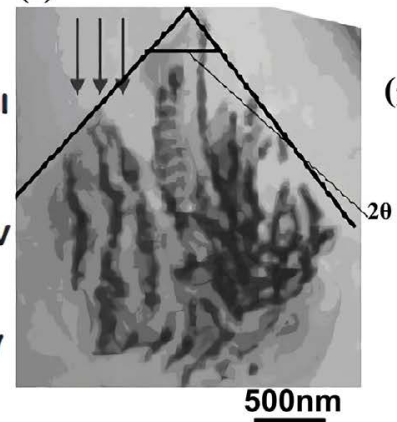

(f)

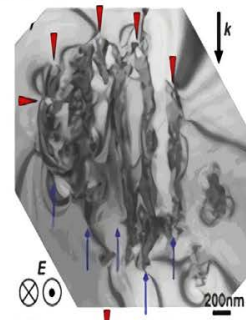

(g)

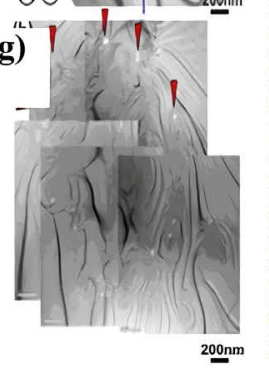

(h)

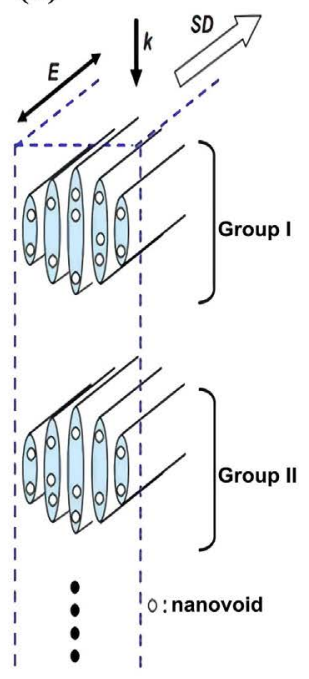

Figure 19. (a) Schematic illustration of the laser irradiated pattern. The light propagation direction (k) and electric field (E) are shown. (b) Optical micrograph of the mechanically thinned sample to show cross sections of laser-irradiated lines (200 nJ/pulse). (c) Bright-field TEM image of the cross section of a line written with pulse energy of $300 \mathrm{~nJ} / \mathrm{pulse}$. (d) Schematic illustration of a geometric relationship between the irradiated line and the cross-sectional micrograph. (e) Magnified image of a rectangular area in (c). Laser-modified layers with a spacing of $150 \mathrm{~nm}$ are indicated by arrows. (f) Bright-field TEM image of a portion of the cross section of a line written with a pulse energy of $200 \mathrm{~nJ} /$ pulse. (g) Zero-loss image of a same area as in ( $\mathrm{f}$ ) with nanovoids appearing as bright areas. Correspondence with (f) is found by noting the arrowheads in both micrographs. (h) Schematic illustrations of the microstructure of a laser modified line. Light-propagation direction (k), electric field (E), and scan direction (SD) are shown. Only two groups (groups I and II) of the laser-modified microstructure are drawn [29] [30].

In this case diffraction processes may be generated in two stages: 1 , formation of diffraction rings of focused beams [7] [8] [9] and second, formation of diffracting gratings in the time of redistribution of second-order Cherenkov radiation [7] [8] [9]. Second case is analogous to the creation of self-diffraction gratings in NLO, but for Figure 19(c) and Figure 19(g) our gratings are limited by Much cone of Cherenkov radiation. Roughly speaking only Figures 19(e)-(g) are represented "clean" breakdown.

Two damages region in a crystal with moderately high density of inclusions were received in [22] for $\mathrm{KCl}$ after irradiation by $\mathrm{CO}_{2}$-laser pulses (wavelength $10.6 \mu \mathrm{m}$, duration of pulse $30 \mathrm{~ns}$ ). The laser was known to be operating in the lowest-order transverse Gaussian mode. There were several longitudinal modes, however, which contributed a time structure to the pulse, periodic at the cavity round-trip time. The phase relationships between the longitudinal modes varied from shot to shot, changing the details of the time structure and causing the peak of the envelope to fluctuate by $\pm 15 \%$ [26]. These results are presented in Figure 20 [31].

Successive laser shot $(1 / \mathrm{sec})$ were focused into bulk single crystals using a 1 -inch focal length "Irtran 2" lens. The breakdown was monitored by observing the visible light from the focal region and by examining the damaged region under the microscope. It was found that most of the crystals suffered some damage 


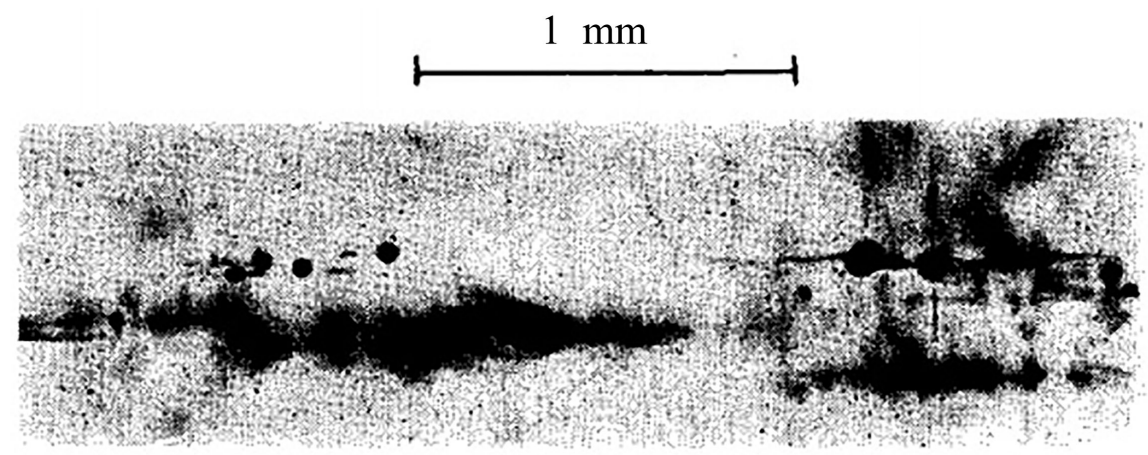

(a)

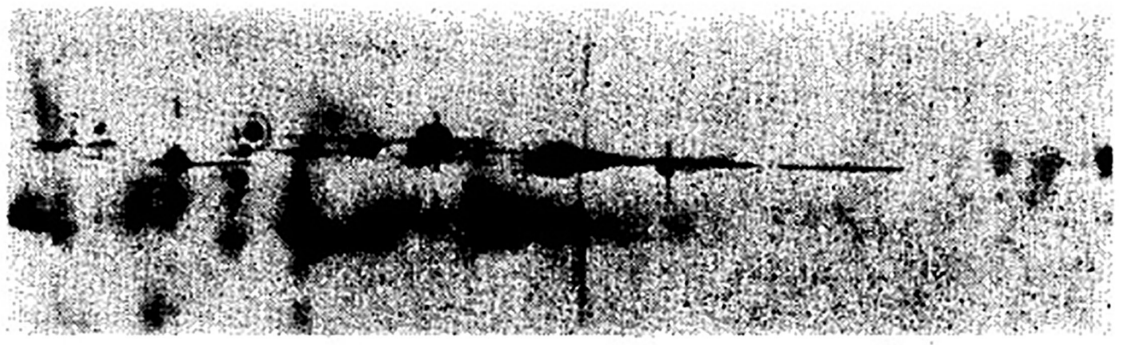

(b)

Figure 20. Two damages regions in a crystal $\mathrm{KCl}$ with moderately high density of inclusions. The round black objects are bubbles. The radiation, incident from left to right, was yust at the intrinsic breakdown threshold. In one case (a) there was damage only at the inclusions. In (b), intrinsic breakdown occurred as evidenced by the pointed bubble. The straight lines represent cleavage [31].

even at relatively low power levels. The thresholds of this type of damages are varied by an order magnitude from one position in the crystal to another. At any particular energy level, damage would occur on the first laser shot or not at all.

Figure 20(a) shows that spatial inhomogeneities are in fact inclusions [31]. The damage bubbles occur randomly near, not necessarily in, the tiny focal volume. At a well-defined power threshold, an elongated pointed bubble forms, its vertex falling at the focus (Figure 20(b)). This power level is regarded ass the bulk intrinsic breakdown threshold. Its value is reproducible in crystals from different manufacturers, with inclusions or without. When no inclusion-free samples of a compound were available, the considerations mentioned above were used to determine the dielectric strength [31].

A ring-shape zone supports major spots initiated by the highest intense defects of the initial beam (depth $z=30 \mathrm{~m}$ ). These "hot" spots self-focus more and more over several meters, while they excite secondary smaller-scaled filaments in their vicinity $(z=35 \mathrm{~m})$ [32]. Evacuation of power excess undergone by the primary filaments finally allows transfer of power to the central zone of the beam, which serves as an energy reservoir for exciting new sequences of small spots ( $z$ $=50 \mathrm{~m}$ ) (Figure 21). Numerical data were represented for duration of pulse 85 fs and power few terawatt [33]. From these results specific geometrical zones in the beam pattern were selected [32]. 

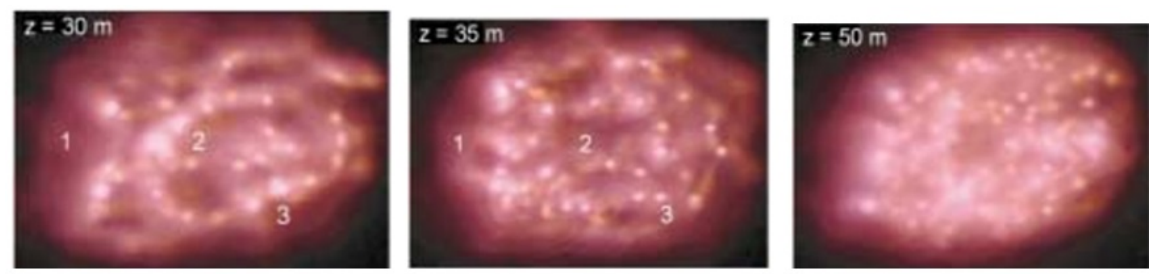

Figure 21. Filamentation pattern of the $700 P_{c r}$ beam delivered by Teramobile laser. Labels 1 - 3 spot specific beam zones commented on in the text [32].

Characteristics examples are indicated by labels $1-3$ [32]:

1) points to a couple of hot couples surviving at further distances;

2) indicates in active region of the beam, where intense filaments decay into cell of lesser intensity;

3) identifies an area including a cross-wise structure that keeps some filaments robust over $5 \mathrm{~m}$.

Condition of receiving self-focusing is next: self-focusing must be more as diffraction [2] [7] [8] [9] [37]. Roughly speaking maxima of diffraction pattern may be represented as traces of optical breakdown.

Other experimental data about laser-induced optical breakdown in matter are represented in [2] [7] [8] [9].

Thermal and plasma models of laser-induced optical breakdown are analyzed in [2] [4].

Now we are presenting direct cascade models of laser-induced optical breakdown according to [7] [8] [9].

The first laser-induced filaments were received in the liquid. Later researches shown that analogous phenomena are generated in solid and gas matter too. Therefore, first models were created for the nonlinear Kerr media and were used for all types of irradiated matter [4] [5]. Strongly speaking, these filaments are sparks of optical breakdown. More universal concept is physical-chemical.

But Kerr media are represented liquids basically. For solid state basic phenomena are laser-induced electrostriction [1] [2] [7] [8] [9]. In the gas case we can have other nonlinear optical phenomena. Therefore, we must select more universal concept for the determination $P_{c r}$. It may be physical-chemical method. In this case we must have concentration of proper centers of scattering (absorption) of laser radiation, which are generated proper nonlinear optical phenomenon, and its activation energy. The self-focusing is nonlinear optical process therefore $P_{c r}$ or the critical value of energy may be determined in next way. Volume density of energy of the creation self-focusing process may be determined with help next formula $W_{\text {crvol }}$ [7] [8] [9]

$$
W_{\text {crvol }}=E_{a} N_{n c},
$$

where $E_{a}$ : energy of activation proper "nonlinear" centers; $N_{n c}$ : their concentration.

Surface density for optical thin may be determined as [7] [8] [9] 


$$
W_{c r s u r}=\frac{W_{c r v o l}}{\alpha},
$$

where $\alpha$ : absorbance index. Integral value of energy may be determined as [7] [8] [9]

$$
W_{\text {crin }}=W_{\text {crsur }} \cdot S,
$$

where $S$ : the square of irradiation.

In this case [7] [8] [9]

$$
P_{c r}=\frac{W_{c r i n}}{\tau_{i r}},
$$

where $\tau_{i r}$ is duration of laser irradiation.

The determination the concentration of scattering centers must be determined with conditions of proper experiment. It is determined by the conditions of observation the proper phenomena.

Next step of determination the density of energy in our cascade is condition of diffractive stratification. This condition may be determined with help of sizes the diffractive rings. We can estimate density of energy in plane of creation the diffractive stratification for $n=5$.

The explanation of creation the laser-induced filaments have various interpretation. Firstly [7] [8] [9] is the creation wave-guide zones after point of collapse. In this case filaments have little life-time.

Conic part of filament radiation has continuum spectrum: from ultraviolet to infrared. At first this effect was called superbroadening. Therefore, it may be interpreted as laser-induced Cherenkov radiation [7] [8] [9]. The angle $2 \theta$ in the vertex of an angle of Figure 19(e) is double Cherenkov angle. In this case we have frozen picture of laser-induced destruction of $4 \mathrm{H}-\mathrm{SiC}$ with help Cherenkov radiation [7] [8] [9].

The Cherenkov radiation is characterized by two peculiarities [7] [8] [9] [34] [35] [36]: 1) creation of heterogeneous shock polarization of matter and, 2) radiation of this polarization. The methods of receiving shock polarization may be various: irradiation by electrons, $\gamma$-radiation, ions and excitation with help pulse fields. The stratification of this radiation on other type's radiation (volume, pseudo-Cherenkov a.o.) has relative character and may be represented as laser-induced Cherenkov radiation. Therefore in future we'll be represented conical part of filament radiation as Cherenkov.

This fact may be certified with macroscopic and microscopic ways.

First, macroscopic may be represented according to [34]. The similarity between charge particle and light-induced Cherenkov radiation one can invoke the analogy between Snell's law and Cherenkov radiation Figure 22) [34].

This natural since both effects can be derived in the same way from the Huygens interference principle. In [34] the point of intersection of a light pulse impinging at an angle $\varphi$ on a boundary between two media moves with velocity $V=\frac{C}{n_{1} \cos \varphi}$. This relation with Snell's law, gives the Cherenkov ratio [34]. 


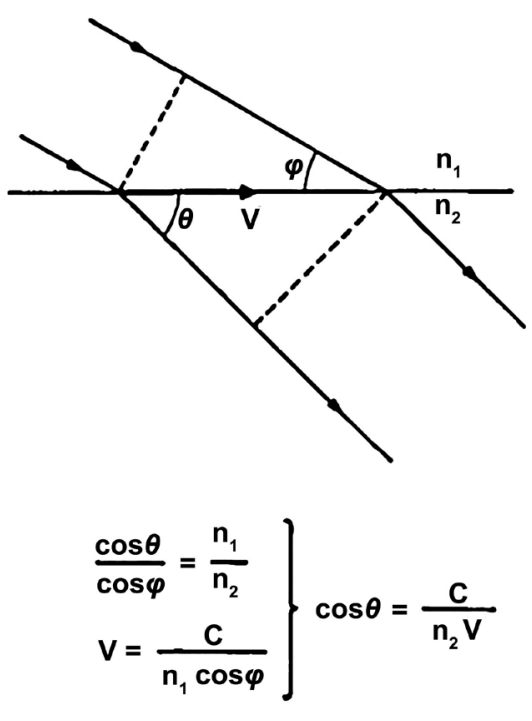

(a)

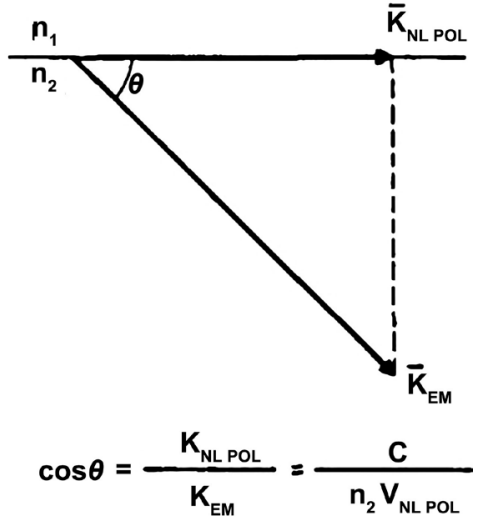

(b)

Figure 22. (a) Analogy between Snell's law and Cherenkov radiation. The point of intersection of a light pulse impinging upon a boundary two media moves with velocity $V=$ $C / \cos \varphi$ Combining this relation with Snell's law one obtains the Cherenkov relation, $\cos \theta$ $=C / n_{2} V$. (b) The Cherenkov angle relation can be obtained from the conservation of the longitudinal component of a linear momentum at a boundary between media along which a nonlinear polarization is propagated [34].

$$
\cos \theta=\frac{C}{n_{2}(\omega) V}
$$

This formula allows explaining the angle differences for various types of Cherenkov radiation. In this case $V$ may be represented as velocity of generation of the optical-induced polarization too [3] [5].

Thus the refraction law a light at the boundary between two media is the same as the condition for Cherenkov emission by a source moving along the boundary. In nonlinear medium the emitted frequencies may be differed from the excitation frequency. The Cherenkov relation is still valid since the constructive interference occurs at a given Cherenkov angle for each Fourier frequency component of the light-induced nonlinear polarization. In a sense, one can speak about a nonlinear Snell-Cherenkov effect [34].

The microscopic mechanism of laser-induced Cherenkov radiation is expansion and application of Niels and Aage Bohrs microscopic theory of Cherenkov radiation as part of deceleration radiation on optical case [7] [8] [9] [36]. For optical case the Bohrs hyperboloid must be changed on Gaussian distribution of light for mode $\mathrm{TEM}_{00}$ or distribution for focused light of laser beam [7] [8] [9]. In this case Cherenkov angle may be determined from next formula

$$
\theta_{C h}+\alpha_{i r}=\frac{\pi}{2} \text { or } \theta_{C h}=\frac{\pi}{2}-\alpha_{i r},
$$

where $\alpha_{i r}$ angle between tangent line and direction of laser beam. 
Angle $\alpha_{i r}$ was determined from next formula [7] [8] [9]

$$
\tan \alpha_{i r}=\frac{d_{b}}{l_{s f}},
$$

where $d_{b}$ diameter of laser beam, $(7 \mathrm{~mm}), 1_{s f}$ length of self-focusing. In our case $\alpha_{i r}$ is angle of self-focusing.

This formula is approximate for average angle $\alpha_{i r}$.

The Golub formula (11) was used for the determination product $n_{2}(\omega) V_{n l \text { pol }}$ [7] [8] [9]. Self-focusing and Cherenkov angles and product $n_{2}(\omega) V_{n l \text { pol }}$ were estimated for $\mathrm{LiF}, \mathrm{CaF}_{2}$, fused silica, water and glass BK-7 in [9].

Thereby microscopic modified Bohrs theory and macroscopic Golub model are mutually complementary methods [7] [8] [9].

The decreasing of Cherenkov angle and product $n_{2}(\omega) V$ for increasing of laser radiation intensity are corresponded to increasing of nonlinear refractive index and decreasing of velocity of polarization (multiphoton and multiwave processes) [7] [8] [9].

In whole microscopic mechanism of laser-induced Cherenkov radiation may be represented as nonequilibrium spectrum of all possible Nonlinear Optical phenomena in the local points of propagation of the laser beam. It may be Raman and Brillouin scattering, up- and down-conversion, generation of harmonics and various interference of these processes and phenomena, which are generated the continuous spectrum from ultraviolet to infrared regions [2] [7] [8] [9] [34].

The estimation of sizes of the cascade of volume destructions of Figure 19(c) may be explained in next way [7] [8] [9]. The sizes (diameters) of proper stages $d_{n i r}$ of cascade are proportionally to corresponding diffraction diameters $d_{\text {ndif }}$

$$
d_{\text {nir }}=k d_{\text {ndif }},
$$

where $k$ is the proportionality constant.

The diffraction diameters $d_{\text {ndif }}$ may be determined with help condition of diffraction-pattern lobes (modified Rayleygh ratio)

$$
d_{\text {ndif }}=n \lambda .
$$

The estimations of first five diffraction diameters $d_{\text {ndif }}$ for $\lambda=800 \mathrm{~nm}$ were represented in [7] [8] [9].

The distance between diffraction spots and proper moving foci may be determined with help next formula

$$
l_{n f}=\frac{d_{n d i f}}{2 \tan \frac{\varphi}{2}} .
$$

These distances for $\varphi_{1}=20^{\circ}$ and $\varphi_{2}=30^{\circ}$ were represented in [7] [8] [9]. In general case the angle $\varphi$ is depended from homogeneity of irradiated matter or intensity of irradiation.

Qualitative explanation of development of cascade the destructions may be next. The focus of each diffraction zone (spot) is the founder proper shock opti- 
cal breakdown. But foci with more high number may place in the "zone" of influence of previous foci. Therefore, only first stage of Figure 19(c) is represented pure shock mechanism (Mach cone). Mach cones are characterized the second and third stages of Figure 19(c). But its maximums are displaced from center. It may be result if interaction second and third shock waves with previous shock waves: first for second wave and first and second for third wave. The chock mechanism of destruction certifies a linear direction of optical breakdown. This direction is parallel to direction of shock wave and radiated spectrum is continuum as for Cherenkov radiation and as for observed laser-induced filaments in water and air [7] [8] [9]. Thus basic creator of optical breakdown traces is secondary Cherenkov radiation and shock waves. This radiation is absorbed more effectively as laser radiation and therefore the creation of optical breakdown traces is more effectively as for beginning laser radiation. Cherenkov radiation is laid in self-absorption range of $4 \mathrm{H}-\mathrm{SiC}$, but $800 \mathrm{~nm}$ radiation - in intrinsic range. For the testing of this hypothesis we must measure the spectrum of secondary radiation. In this case we can use physical-chemical cascade model of excitation the proper chemical bonds of irradiated matter in the regime of saturation the excitation.

The conclusion about diffractive stratification of focused radiation may be certified by experimental data of Figure 19(c) [7] [8] [9].

These results are corresponded to Lugovoy-Prokhorov theory [37] too: distance between contiguous elements is smaller as distance between microscopy ocular and first stage of cascade (correlation of this distance is proportional to $\lambda / d$ ) but distance between contiguous elements of cascade is equal and proportional to half wavelength [7] [8] [9].

Cherenkov radiation has next peculiarity. We know fact that induced radiators give possibility to receive two coherent light sources. This fact is used for the standard interference [34] [35] [36]. Analogous phenomenon must be observed for Cherenkov radiation too [7] [8] [9] [35]. If we transmit light beam through two little volumes of similar matter, then we receive two coherent sources. This property is characterized for any wavelength of continuous Cherenkov spectrum [35]. Therefore, interference for Cherenkov radiation have broad spectral region [35].

Qualitative explanation of development of cascade the destructions (Figure 19(c)) may be next [7] [8] [9]. The focus of each diffraction zone (spot) is the founder proper shock optical breakdown. But foci with more high number may placed in the "zone" of influence of previous foci. Therefore, only first stage of Figure 19(c) is represented pure shock mechanism (Mach cone). Mach cones are characterized the second and third stages of Figure 19(c). But its maximums are displaced from center. It may be result if interaction second and third shock waves with previous shock waves: first for second wave and first and second for third wave. The chock mechanism of destruction certifies a linear direction of optical breakdown. This direction is parallel to direction of shock wave and ra- 
diated spectrum is continuum as for Cherenkov radiation and as for observed laser-induced filaments in water and air [7] [8] [9]. Thus basic creator of optical breakdown traces is secondary Cherenkov radiation and shock waves. This radiation is absorbed more effectively as laser radiation and therefore the creation of optical breakdown traces is more effectively as for beginning laser radiation. Cherenkov radiation is laid in self-absorption range of $4 \mathrm{H}-\mathrm{SiC}$, but 800 $\mathrm{nm}$ radiation in intrinsic range [7] [8] [9]. For the testing of this hypothesis we must measure the spectrum of secondary radiation. In this case we can use physical-chemical cascade model of excitation the proper chemical bonds of irradiated matter in the regime of saturation the excitation.

We can rough estimate basic peculiarities of energy distribution in Mach cone may be used next formula [7] [8] [9]

$$
E_{1 o b}=\frac{\pi^{2}}{4}\left(\sum_{i=1}^{5} n_{\text {iav }}^{2} l_{\text {iav }}\right) r^{2} N_{a S i C} E_{\text {Zth }},
$$

where $n_{i a r}$ : average visible number of filaments in proper group of cascade, $l_{i a v}=$ $1000 \mathrm{~nm}$, average length of filaments in proper group of cascade, $r=10 \mathrm{~nm}$, average radius of filament, $N_{a}$ : atom density of $4 \mathrm{H}-\mathrm{SiC}$.

The atom density of $4 \mathrm{H}-\mathrm{SiC}$ may be determined with help next formula [7] [8] [9].

$$
N_{a}=\frac{n \rho N_{A}}{A},
$$

where $\rho$ : density of semiconductor, $N_{A}$ : Avogadro number, $A$ : a weight of one gram-molecule, $n$ : number of atoms in molecule. For $4 \mathrm{H}-\mathrm{SiC}$

$N_{a \mathrm{SiC}}=9.4 \times 10^{21} \mathrm{~cm}^{-3}$.

For further estimation we use next approximation $n_{1 a v}=n_{2 a v}=n_{3 a v}=n_{4 a v}=n_{5 a v}=100$, (see Figure 19(c)).

Energy, which is necessary for the optical breakdown our nanotubes may be determined in next way. Zeitz threshold energy for $4 \mathrm{H}-\mathrm{SiC}$ is equaled $E_{Z t h} \sim 25$ $\mathrm{eV}$ [7] [8] [9] [20]. Let this value is corresponded to energy of optical breakdown. Therefore summary energy $E_{10 b}$ is equaled

$$
E_{10 b}=N_{\text {asnt }} \cdot E_{\text {Zth }}=23.2 \mathrm{~nJ} \text {. }
$$

This value is equaled of $\sim 8 \%$ from pulse energy or $\sim 30 \%$ from the effective absorbed energy of pulse. In this case we have more high efficiency of transformation initial radiation to "irreversible" part of Cherenkov radiation. It is result of more intensive excitation comparatively with classical methods of receiving the Cherenkov radiation. In this case we have pure photochemical processes. The experimental data for intrinsic absorption (Figure 19) show that for short pulse regime of irradiation (femtosecond regime) basic processes of destruction the fused silica and calcium fluoride are photochemical (multiphoton absorption in the regime of saturation the excitation). But basic peculiarity of experimental data Figure 5 is transformation of the initial laser radiation (wavelength 800 $\mathrm{nm}$ ) to continuum Cherenkov radiation. From length of optical breakdown in 
$4 \mathrm{H}-\mathrm{SiC}$ we can determine average absorption index of Cherenkov radiation. It is $\sim 10^{4} \mathrm{~cm}^{-1}$. This value is corresponded to violet-blue range of absorption spectrum of $4 \mathrm{H}-\mathrm{SiC}$ [7] [8] [9].

The difference between generations of surface continuum radiation [7] [8] [9] and optical-induced Cherenkov radiation is next. At first time we have collective electromagnetic processes, which are may be represented as processes with velocity less as phase speed of light in media. Mainly, it is wave processes. In the case of Cherenkov radiation we have directed quantum optical processes, which can represent as processes with velocity more as phase speed of light in matter. Roughly speaking last processes may be had velocity less as phase speed of light in media but it must be local (quantum) [34]. But in this case we must determine the new phase speed of light as speed of collectivization of electromagnetic oscillations for corresponding frequency in irradiated media because we have continuum spectrum of irradiation. In this case the summary speed of interaction light and matter is determined the summary time of corresponding chain of direct optical processes.

Cherenkov radiation may be represented as back process of Nonlinear Optics too. Roughly speaking Nonlinear Optics is optics of nonlinear polarization. But intense laser irradiation is generated nonlinear polarization and Cherenkov radiation [7] [8] [9] [34]. Therefore these processes have identical nature [7] [8] [9] [34].

We can estimate chain of critical value of energy for the $4 \mathrm{H}-\mathrm{SiC}$ from physical-chemical point of view, too.

Critical value of energy, which is necessary for the beginning of self-focusing, may be determined in next way. Volume density of energy of the creation self-focusing process may be determined with help formula (19). In further we made next approximation [7] [8] [9]: $E_{a}=h v=1.5 \mathrm{eV} ; N_{n c}=\left(10^{14}-10^{16}\right) \mathrm{cm}^{-3}$. Then we have for SiC $W_{\text {crvol }}=2.4 \times\left(10^{-5}-10^{-3}\right) \mathrm{J} \cdot \mathrm{cm}^{-3}$. For $\operatorname{SiC} \alpha=0.1 \mathrm{~cm}^{-1}$. And $W_{\text {crsur }}=2.4 \times\left(10^{-54}-10^{-2}\right) \mathrm{J} \cdot \mathrm{cm}^{-2}$.

Integral value of energy may be determined according by formula (36). For Figure 19 (c) for $r=2 \mu \mathrm{m}, S=1.256 \times 10^{-7} \mathrm{~cm}^{2}$. Therefore $W_{\text {crin }}=3 \times\left(10^{-11}-10^{-9}\right) \mathrm{J}$. For $r=1 \mathrm{~mm}$ we have $W_{\text {crin }}=1.9 \times\left(10^{-6}-10^{-4}\right) \mathrm{J}$.

These estimations are corresponded to estimations, which are received with help formulas for Kerr media. Roughly speaking they are equivalence [7] [8] [9]. For the gases this method allows to estimate the energy of its optical breakdown.

Next step of determination the density of energy in our cascade is condition of diffractive stratification. This condition may be determined with help of sizes the diffractive rings. We can estimate density of energy in plane of creation the diffractive stratification for $n=5$.

Maximum diameter of diffractive pattern is determined for fifth diffractive ring. For this case average density of energy in plane of diffractive rings is equaled.

We can estimate corresponding correlation between energies for the next 
processes: laser irradiation, diffractive stratification, Cherenkov radiation and optical breakdown [7] [8] [9].

A density of laser irradiation is equaled

$$
W_{\text {avdr }}=\frac{E_{p}}{S} .
$$

where $E_{p}$ : energy of laser pulse. For $E_{p}=200 \mathrm{~nJ}$ and $E_{p}=300 \mathrm{~nJ}$ and $S=1.256 \times$ $10^{-7} \mathrm{~cm}^{2}$ we have next value of $W_{\text {avdr }} 1.6 \mathrm{~J} / \mathrm{cm}^{2}$ and $2.4 \mathrm{~J} / \mathrm{cm}^{2}$. If we multiple these value of the absorbance index of $\operatorname{SiC} \alpha=0.1 \mathrm{~cm}^{-1}$ then we are receiving the volume density of energy $W_{\text {avdrol }} 0.16 \mathrm{~J} / \mathrm{cm}^{3}$ and $0.24 \mathrm{~J} / \mathrm{cm}^{3}$. Really value is 0.4 from represented data (reflectance is 0.6) and are 0.064 and $0.096 \mathrm{~J} / \mathrm{cm}^{3}$ [7] [8] [9].

Correlation $W_{\text {avdrvol }} / W_{\text {crvol }}$ for real values for $\mathrm{SiC}$ is equaled from 27 to 2700 .

Density of energy of optical breakdown $W_{o b}$ for $\mathrm{SiC}$ is equaled $18,800 \mathrm{~J} / \mathrm{cm}^{3}$. Therefore correlation $W_{\text {avdrool }} / W_{\text {crvol }}$ is equaled 78,333 and 117,500 [7] [8] [9].

The analogous chain processes may be mage for other media ( $\mathrm{LiF}$, water, hard water, $\mathrm{CaF}_{2}, \mathrm{NaCl}, \mathrm{CS}_{2}, \mathrm{CCl}_{4}, \mathrm{C}_{2} \mathrm{HCl}_{3}$ and fused silica) [9].

Concept of diffractive stratification allows explaining the surface character of Cherenkov radiation. This radiation is generated in the region of corresponding focused diffractive ring [7] [8] [9].

Rayleygh model is resolution of Besant problem [38], which is formulated in next form "An infinite mass of homogeneous incompressible fluid acted upon by no forces is at rest, and a spherical portion of the fluid is suddenly annihilated; it is required to find the instantaneous alteration of pressure at any point of the mass, and the time in which the cavity will be fill up, the pressure at a infinite distance being supposed to remain constant."

Rayleygh received resolution this problem for the sound shock processes for liquid. In this case cavitations' bubbles have sizes from a few millimeters to a few centimeters [7] [8] [9] [38] [39].

In our case (Figure 19(h)) sizes of our nanovoids are equaled $15-20 \mathrm{~nm}$. Therefore we must change "sound" mechanism of creation cavitations bubbles on electromagnetic. This problem was resolved with help change speed of sound or speed of light.

The sizes of nanovoids (Figure 19(h)) may be determined with help modified Rayleygh model [7] [8] [9] and its form, the help methods of continuum mechanics. [7] [8] [9] in next way.

Nanovoids may be represented as results of the laser-induced laser-induce breakdown and creation of cavitations bubbles [7] [8] [9] too. The light pressure may be determined with help of next formula [24]

$$
p_{0}=\frac{E_{i r}}{\tau_{i} c S},
$$

where $E$ : energy of irradiation, $\tau_{i}$ : pulse duration, $S$ : area of irradiation zone, $c$. speed of light. For circle symmetry 


$$
S=\pi r^{2}
$$

where $r$. radius of laser spot.

For the estimations of maximal radius of nanovoids we must use modified Rayleygh formula [7] [8] [9]

$$
R_{\max }=\frac{2 R}{0.915 r} \sqrt{\frac{E_{i r}}{\pi \tau_{i} c E}}
$$

where $T_{c}$ : the time of creation the nanovoid (bubble), $R$ is radius of nanovoid, $r$. radius of irradiated zone, $E$ : Young module, $E_{i r}$ : energy of one pulse. $\tau_{i}$ : duration of pulse [7] [8] [9].

If we substitute $r=250 \mathrm{~nm}, R=10 \mathrm{~nm}, E=600 \mathrm{GPa}$ [4] [6], $E_{i r}=300 \mathrm{~nJ}, \tau_{i}=$ $130 \mathrm{ps}, c=3 \times 10^{8} \mathrm{~m} / \mathrm{s}$, than have $R_{\max }=11 \mathrm{~nm}$.

The speed of shock waves for femtosecond regime of irradiation is less as speed of sound. But we have two speeds of sound in elastic body: longitudinal $\vartheta_{l s}$ and transversal $\vartheta_{t s}$ [3]. Its values are determined with next formulas

$$
\vartheta_{l s}=\sqrt{\frac{E(1-v)}{\rho_{o}(1+v)(1-2 v)}} \text {, and } \vartheta_{t s}=\sqrt{\frac{E}{2 \rho_{o}(1+v)}},
$$

where v. Poisson's ratio [7] [8] [9]. The ratio between of these two speeds is equaled

$$
\alpha=\frac{\vartheta_{t s}}{\vartheta_{l s}}=\sqrt{\frac{(1-2 v)}{2(1-v)}} .
$$

But this ratio must be true for shock waves too. Therefore, for silicon carbide for $v=0.45$ [7] [8] [9] $\alpha=0.33$. Roughly speaking last ratio is determined the step of ellipsoidal forms of our nanovoids (Figure 19(h)).

In [7] [8] [9] allow estimating maximal longitudinal and transversal $R_{\max i}, i \in(l, t)$. These values are $6 \mathrm{~nm}$ and $19 \mathrm{~nm}$ properly.

In this case we represented $4 \mathrm{H}-\mathrm{SiC}$ as isotropic plastic body. For real picture we must represent hexagonal structure. But for the qualitative explanation of experimental data of Figure 19(h) this modified Rayleygh model allows explaining and estimating the sizes and forms of receiving nanovoids [7] [8] [9].

As we see, for laser-induced breakdown we must include self-focusing processes too. The problem of creation initial inoculating concentration of electrons is one of main problems Nonlinear Optics too. Therefore we must include in the problem of optical breakdown the heterogeneity materials and heterogeneity of interaction light and matter, including diffraction stratification, generation of continuum radiation (including Cherenkov radiation), interference Cherenkov radiation and direct optical breakdown. These addition factors allow explaining basic peculiarities of interaction laser irradiation and matter, including gases (Figure 21), liquid (Figure 18) and solid (Figure 19 and Figure 20).

But for more long time of irradiation we have second-order processes of disorder radiation, including reradiation and reabsorption [3]. In this case we may be having processes of heating and creation of plasma clouds [2] [4]. Foe shorter 
regime irradiation a probability of cascade step-by-step laser-induced direct multiphoton excitation is increased and therefore we have third scenario of these processes [7] [8] [9].

Thus, methods of Relaxed Optics allow integrating processes of radiated and nonradiated relaxation (Nonlinear and Relaxed Optics) of first-order optical excitation in one system and allow explaining processes of laser-induced optical breakdown and shock processes with one point of view. For qualitative explanation of corresponding experimental data, we must add using methods by physical-chemical models and methods of diffraction stratification and laser-induced Cherenkov radiation [7] [8] [9].

Laser-induced shock processes have specific peculiarities. In general case we have electromagnetic and acoustic shock processes [7] [8] [9]. Formally these processes have similar nature.

Speed of electromagnetic shock processes (speed of polarization the media in the result of corresponding interaction) must be more as phase speed of light in media. In this case phase speed of light in media has next physical nature: it is speed of collectivization the electromagnetic oscillations for proper frequency. Roughly speaking it is electromagnetic characteristic of media, which is corresponded to its electron and ion subsystems. Example of this type process is Cherenkov radiation. In this case we have radiated reaction of media on heterogeneity excitation of media in shock regimes of interactions.

Speed of acoustic shock processes (speed of motion proper object in media) must more as speed of sound in media [7] [8] [9]. But speed of sound is media is average heat speed of media, which is connected with atomic structure of media. Examples of these processes are: flight of airplanes or rocket in with supersonic speed; various explosions. Roughly speaking, explosions may be characterized as chemical process with speed more as sound speed in media. Mach number is characterized in this case the macroscopic "detonation" of corresponding process.

Both processes (electromagnetic and acoustic) are characterized by Much cone, which is created by proper vectors of speed processes or object and speed characteristic of media (polarization or sound) [7] [8] [9].

Laser-induced shock processes may be represented as analogous to acoustic explosions. But this processes is realized with electromagnetic speed. In this case we must have "electromagnetic" explosion as Cherenkov radiation [7] [8] [9].

The formation of the laser-induced shock processes may be explained with help concept of coherent structures of Relaxed Optics [1]. In this case generation of proper shock process may be represented as chain of coherent processes of interaction light and matter with electromagnetic speed. In this case summary speed of chain process must be less as phase speed of light in media but speed of each term of this chain must be more as phase speed of light in media. It is basic peculiarity of formation laser-induced shock processes comparatively with other electromagnetic processes. From point of view the kinetic concept of Relaxed Optics [1] we must have multiphoton process in the regime of saturation the ex- 
citation.

In whole the nonequilibrium and irreversible shock processes may be had electromagnetic and acoustic nature [7] [8] [9].

Nature of laser-induced breakdown is determined by speed of energy transfer from laser irradiation to matter. For impurity mechanisms of absorption the laser radiation may be realized next scenario [7] [8] [9]:

1) Multiphoton absorption is comparatively low intensity but time of irradiation is long-run. In this case we have heating of matter in the region of the absorption of laser radiation and thermal mechanism of optical breakdown. Other words absorptive energy may be represented as heat, which is caused the heating of media and its thermal breakdown. The process is characterized the speed of sound in matter and time of creation the heating of irradiated material is equaled $\sim\left(10^{2}-10^{3}\right) \tau_{i}$ for millisecond and nanosecond regimes of irradiation [7] [8] [9].

2) Multiphoton absorption is higher as in first case, but it may be source of other collective physical phenomena: generation of plasma, which may be used for the breakdown of irradiated matter. It is plasma mechanism of optical breakdown. Time of formation the plasma in irradiate matter is lesser and roughly speaking it determines the speed of light. In whole the speed of this process is lesser as phase speed of light in irradiated matter. But this time is depending from intensity of irradiation too [7] [8] [9].

3) Multiphoton absorption is higher as in case 2 and may be source of direct optical breakdown. But this process must have speed more as phase speed of light in media [7] [8] [9].

But laser-induced optical breakdown is possible only in volume of irradiated matter. On surface we will be had ablation for thermal and plasma mechanisms of laser-induced optical breakdown and sublimation for plasma and direct mechanisms of optical breakdown.

Now we used physical-chemical method of estimation for the modeling experimental data for $\mathrm{KCl}$ (Figure 20). Density of atoms of $\mathrm{KCl}$ was determined with help formula (1) and it equals $3.1 \times 10^{22} \mathrm{~cm}^{-3}$. Zeits energy for $\mathrm{KCl}$ has value $\sim 30$ $\mathrm{eV}[20]$.

We used next approximations. Photography of Figure 20 gives a blurry image compared to the bright-field TEM image of Figure 19. Therefore, we can't see the microstructure of optical breakdown. But we use rough average approximations for diameter $d_{\text {average }}$ and length $l$ of cascade laser-induced optical breakdown of Figure 6. Volume of cascade was determined as cylinder volume.

Figure 20 is similar to Figure 19(c). But regimes of irradiation of Figure 20 are similar to mode $\mathrm{TEM}_{01}$. Therefore, we have two channels of generation the cascade of laser-induced optical breakdown [7].

The distances between bubbles of Figure 20(b) are more as between regions of destruction of Figure 19(c). But conditions of focusing the radiation in these both cases are equivalence. Therefore, the distances between neighboring bub- 
bles $l_{2}$ of Figure 20(b) and neighboring regions of destruction $l_{1}$ of Figure 19(c) are connected by next formula

$$
l_{2}=\frac{d_{\text {ndif } 2} \tan \left(\frac{\varphi_{1}}{2}\right)}{d_{\text {ndif } 2} \tan \left(\frac{\varphi_{2}}{2}\right)} l_{1}=\frac{\lambda_{2} \tan \left(\frac{\varphi_{1}}{2}\right)}{\lambda_{2} \tan \left(\frac{\varphi_{2}}{2}\right)} l_{1} .
$$

In whole the correlation of this distance is depended from wavelength of irradiation and focusing angles, including intensity of irradiation? Which is determined the step of homogeneity of irradiated matter. If we substitute in formula (53) $\lambda_{2}=10.6 \mu \mathrm{m}$ and $\lambda_{1}=0.8 \mu \mathrm{m}$ and $\varphi_{1}=\varphi_{2}$ then we'll receive

$$
l_{2}=13.25 l_{1} \text {. }
$$

Energy characteristics of irradiation weren't represented in [31]. Therefore we select value $2 \mathrm{~J} /$ pulse from [7]. In this case we have effective using energy. Methods of estimations of energy characteristics for $\mathrm{KCl}$ are rougher as for $4 \mathrm{H}-\mathrm{SiC}$. But we must suppose that focused laser irradiation has diffraction stratification, generation of Cherenkov radiation and interference of this Cherenkov radiation. On Figure 20(b) 5 - 7 steps of cascade optical breakdown we see. Sources of Cherenkov radiation id diffraction stratified cones [7].

If this scenario is true we have as for $4 \mathrm{H}-\mathrm{SiC}$ effective transformation the energy of laser radiation to cascade of laser-induced breakdown for $\mathrm{KCl}$ too. This value is 11.6 - 17.4 percents.

Experimental data, which are represented in Figure 19 and Figure 20, are similar to bead lightning [7] and resemble a frozen picture in a travelling wave lamp [7]. But for the formation these processes we must have two electrodes and modulated external field. For the case of laser-induced breakdown we have only laser field, its nonlinear transformation, including diffractive stratification of laser beam, a generation of Cherenkov radiation and its interference, and multiphoton absorption. In this case we have inner nonlinear and relaxed optical processes.

As we see basic mechanism the inhomogeneities cascade damages of laser-induced optical breakdown is nonlinear optical transformation of initial radiation. Inclusions in $4 \mathrm{H}-\mathrm{SiC}$ and $\mathrm{KCl}$ are distributed homogenously and therefore one can't be the source of laser-induced inhomogeneities.

\section{Some Technological Applications}

\subsection{Annealing of Radiation Damages}

Now we'll show applications of laser radiation for the annealing the damages of ion implantation [1] [6] and nuclear reactor neutron radiation [9].

The results of the positive resolution the problem of laser annealing of ion-implanted InSb and InAs were represented. The conclusion of previous chapter may be next: the laser annealing of these semiconductors the ruby laser is impossible. For more precision research of this hypothesis the ruby laser (du- 
ration of pulse $20 \mathrm{~ns}$, wavelength $0.6943 \mu \mathrm{m}$ ) and $\mathrm{CO}_{2}$-laser (pulsing with duration of pulse $10 \mu \mathrm{s}$, and stationary, wavelength $10.6 \mu \mathrm{m}$ ) irradiation the $\mathrm{Mg}^{+} / \mathrm{InSb}$ (Mg ion energy $100 \mathrm{keV}$, dose of implanation $6 \times 10^{14} \mathrm{~cm}^{-2}$ ), $\mathrm{Mg}^{2+} / \mathrm{InSb}\left(\mathrm{Mg}\right.$ ion energy $50 \mathrm{keV}$, dose of implanation $\left.6 \times 10^{14} \mathrm{~cm}^{-2}\right)$ and $\mathrm{S}^{+} / \mathrm{InAs}$ (S ion energy $40 \mathrm{keV}$, dose of implanation $\times 10^{15} \mathrm{~cm}^{-2}$ ) were provided. For observation method of measurement Hall concentration of charge with layer-by-layer etching and method of Rutherford backscattering ions of He with energy of $1.8 \mathrm{Mev}$ and protons with energy $500 \mathrm{keV}$ were used. The results of integral researches are represented in Figure 23 [1] [6].

The residual defectiveness $\chi_{D}$ of ion-implanted layers is determined as follows

$$
\chi_{D}=\frac{N_{i}-N_{o}}{N_{r}-N_{o}},
$$

where $N_{i}, N_{r}$ and $N_{o}$ : the number of counts of the recording equipment, which corresponds to the number of backscattered helium ions or protons for an ion-implanted sample, an unimplanted sample in random mode and in channeling mode, respectively.

The curves 1, 2 and 3 in Figure 23 are correlated. It is explained as fact of the addition optical damages to radiation defects for intensity of irradiation $0.04 \div$ $0.1 \mathrm{~J} \cdot \mathrm{cm}^{-2}$. Further increasing the intensity to provide to the interaction with optical and radiation damages, but in this case the more important role has heating phenomena. For more small intensity of the irradiation $\left(I_{0}<0.04 \mathrm{~J} \cdot \mathrm{cm}^{-2}\right)$

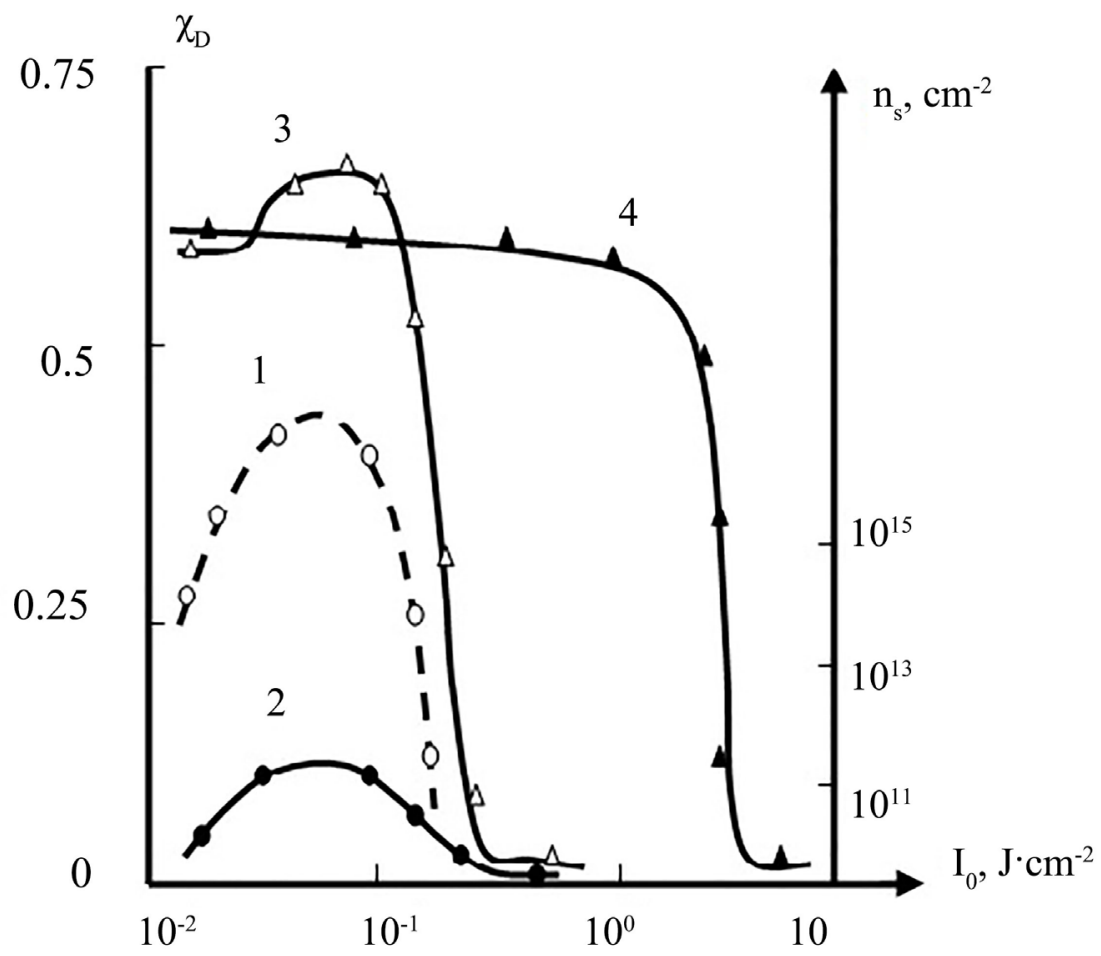

Figure 23. Laser effects in InSb and $\mathrm{Mg}^{2+} / \mathrm{InSb}: 1$ : the energetic dependence of the creation the donor centers in InSb (points are experimental data); $2: \chi_{D}=f\left(I_{0}\right)$ in InSb; 3, 4: $\chi_{D}=f\left(I_{0}\right)$ in $\mathrm{Mg}^{++} / \mathrm{InSb}$ after ruby laser (3) and $\mathrm{CO}_{2}$-laser (4) irradiation [1] [6]. 
the level of the defective state isn't increased. The comparative analysis these data with results of Ruby and Nd lasers irradiation show why laser annealing is impossible for these cases. In this case the full activation the implanted impurity isn't existed for all possible regimes of the irradiation.

For the application for laser annealing $\mathrm{CO}_{2}$-laser $\left(E_{a} \leq h v \leq E_{g}\right)$ the activation of implanted impurity was attained. The radiation defects are annealed for all regimes of the irradiation (stationary and impulsive). The results of the application of the impulse $\mathrm{CO}_{2}$-laser for annealing $\mathrm{Mg}^{2+} / \mathrm{InSb}$ are represented on curve 4 Figure 23. The increasing of the level of the defective state as for curve 3 is absented. Analogous results were received in for $\mathrm{Hg}_{x} \mathrm{Cd}_{1-x} \mathrm{Te}$ and $\mathrm{Pb}_{x} \mathrm{Sn}_{1-x} \mathrm{Te}$ [1] [6]. The correlation between thresholds the melting and the heat destruction of the InSb for Ruby-laser and $\mathrm{CO}_{2}$-laser irradiation were 1:10 and 1:3 respectively. The explanation of this fact is next. For Ruby-laser $\left(h v>E_{g}\right)$ process is provided on succession the creation of optical damages, heating, including reconstruction and annealing primary damages and creation secondary heating defects, melting - destruction. For $\mathrm{CO}_{2}$-laser this succession is more shortly: heating - melting destruction. Coefficient of the creation damages of two-photon absorption is small. In the case of laser annealing of ion-implanted layers, the index of the absorption of ion-implanted layer is $10^{4} \mathrm{~cm}^{-1}$ thus as for pure materials one is 10 $\mathrm{cm}^{-1}$. For comparison, index of the own absorption for ruby laser is $2 \times 10^{5}$ $\mathrm{cm}^{-1}$. Thus for $\mathrm{CO}_{2}$-laser annealing the process has next succession photostimulated and thermal annealing. Both processes (quantum and temperature) have one direction. It is corresponded the independence of this experimental data of time irradiation for this process. In our researches the processes of the melting after $\mathrm{CO}_{2}$-laser irradiation are not observed. The electrophysical properties of laser annealed layers (Volt-Ampere characteristics) are shown the activation of the implanted admixture. For increasing the intensity of the irradiation of stationary laser in 3 - 4 order than for annealing samples are heated to red color and sublimated. In general case the laser irradiation with $h v>E_{g}$ may be used for laser annealing for more long time of irradiation $\left(\sim 10^{-3} s\right.$ and more). For any intensity of the irradiation process of the annealing may be more intensive than processes of the optical and temperature creation of the damages. For this time of the irradiation the concentration of the thermodefects, implanted impurities and optical defects may be correlated for various regimes of the irradiation.

The renewal structure and full activation of the impurity in $\mathrm{Mg}^{+}$-implanted layers InSb is possible only for the $\mathrm{CO}_{2}$-laser irradiation with density of energy flow $I_{0}=10 \mathrm{~J} \cdot \mathrm{cm}^{-2}$ [1] [6]. This effect is integral and isn't depended from the regime of irradiation (impulse or stationary). The renewal of structure is happen after impulse Ruby irradiation with $I_{0}=0.1 \mathrm{~J} \cdot \mathrm{cm}^{-2}$ in measurement Rutherford backscattering spectra ion $\mathrm{H}^{+}$with energy $500 \mathrm{keV}$ and $\mathrm{He}^{+}$with energy 1.8 $\mathrm{MeV}$. But activation of the impurity isn't happening [1] [6].

The renewal structure and full activation of the impurity in $\mathrm{Mg}^{+}$-implanted 
layers InSb (ion energy $100 \mathrm{keV}$, dose $6 \times 10^{14} \mathrm{~cm}^{-2}$ ) is possible only for the $\mathrm{CO}_{2}$-laser irradiation with density of energy flow $I_{0}=10 \mathrm{~J} \cdot \mathrm{cm}^{-2}$ [1] [6]. Analogous process renewal structure and full activation of the impurity in $\mathrm{S}^{+}$-implanted layers InAs (ion energy $40 \mathrm{keV}$, dose $10^{14} \mathrm{~cm}^{-2}$ ) is possible for the $\mathrm{CO}_{2}$-laser irradiation with density of energy flow $I_{0}=12 \mathrm{~J} \cdot \mathrm{cm}^{-2}$ [1].

From the same point of view, the positive results on laser annealing of silicon with neodymium laser radiation are explained. The absorption coefficient of this radiation by a pure material is $60 \mathrm{~cm}^{-1}$, ion-implanted $10^{4} \mathrm{~cm}^{-1}$ [40]. When irradiated with pulses of a ruby laser or radiation of the second harmonic of a neodymium laser, the results are similar to the case of irradiation of ion-implanted layers of indium antimonite and indium arsenide with ruby laser radiation [1] [6] [40].

But the creation of new donor centers in InSb, InAs and Si after pulse laser irradiation with $h v>E_{g}$ allows to receive n-p junctions. n-layers were stable for the $I_{0}=0.1 \mathrm{~J} \cdot \mathrm{cm}^{-2}$ for InSb and $I_{0}=0.16 \mathrm{~J} \cdot \mathrm{cm}^{-2}$ for InAs. Corresponding Volt-Ampere characteristics are curve 2 ( $\mathrm{InSb}$ ) and curve 3 (InAs) on Figure 24. These characteristics have more "bad" character as characteristics of curve 1.

This difference may be explained in the following way. The $\mathrm{CO}_{2}$-laser annealed ion-implanted layers (LAIIL) are the more stable centers as Ruby laser induced donor layers (LIDL). The depth of LAIIL is $0.2-0.4 \mu \mathrm{m}$, the depth of LIDL $0.6-6 \mu \mathrm{m}$. The edge of ion-implanted layer is more abrupt as laser-induced layers. It is basic cause of the best electrophysical properties LAIIL as LIDL.

The difference between physical properties of the LAIIL and LIDL supplement

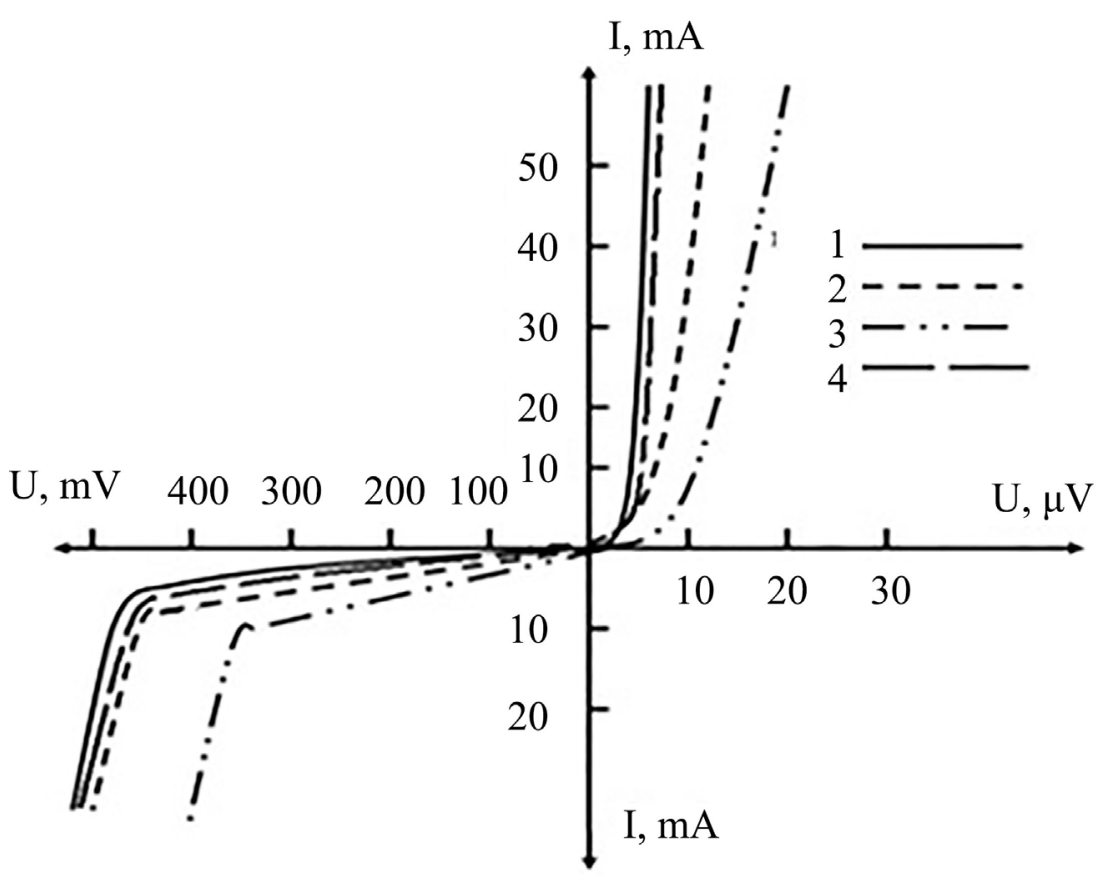

Figure 24. Volt-Ampere characteristics $\mathrm{p}-\mathrm{n}$ and $\mathrm{n}-\mathrm{p}$ junctions: LAIIL, curve $1: \mathrm{Mg}^{2+} / \mathrm{InSb}$ [1] [6], curve 4: S+/InAs [1]; LIDL, curve 2: InSb [1] [6], curve 3: InAs [1] [6]. 
the explanation of the conclusion about impossibility of the laser annealing ion-implanted layers with irradiation in regime $h v>E_{g}$, where $E_{g}$ band gap of semiconductor [1] [6]. The index of absorption in pure $p$-InSb of the $\mathrm{CO}_{2}$-laser irradiation is $10 \mathrm{~cm}^{-1}$, Ruby laser $2 \times 10^{5} \mathrm{~cm}^{-1}$. The index of absorption in $\mathrm{Mg}^{2+} / \mathrm{InSb}$ layers of the $\mathrm{CO}_{2}$-laser irradiation is $10^{4} \mathrm{~cm}^{-1}$. Therefore in the first regime (curve 1, Figure 24) of the irradiation we have one process light scattering on metastable centers and this effect isn't depended from the time of the irradiation. The heat effects (for impulse regime of the irradiation) have the same direction as photoinduced phenomena.

The proper concentration profiles of distribution of donor centers are selected for "stable" regimes of the irradiation for InSb (curve 2 of Figure 24) and InAs (curve 4 of Figure 24).

The difference of absorption indexes for Ruby and $\mathrm{CO}_{2}$-laser irradiation is caused of the impossibility of laser annealing and activation implanted impurity with the help of the laser irradiation with regimes $h v>E_{g}$. For the laser annealing of ion-implanted layers must be used the irradiation with $h v<E_{g}$. The irradiation with $h v>E_{g}$ is the processes of light scattering on stable centers (covalent bonds for InSb and InAs [1] [6]. This process is caused of the generation high concentration of donor centers. Defects of ion implantation have $\mathrm{n}$ type of the conductivity, too. The thermal defects in InSb have p-type of conductivity [1] [6]. Therefore, the contradiction in the explanation of the cause, the creation and annealing damages and activation impurity in InSb is founded on various charge states laser induced damages and thermal defects.

Laser irradiation may be used for the annealing the neutron-induced radiation defects in InSb and InAs magnetic sensors of nuclear reactors [9]. Unlike ion implantation and laser surface doping, these are volumetric effects.

Periodic illumination of magnetic sensors with $\mathrm{CO}_{2}$-laser radiation can practically increase the service life of the sensors until the time of accumulation of decay products of the initial material up to a concentration of $10^{17}-10^{18} \mathrm{~cm}^{-3}$ [9]. For the bulk blistering we must have proton dose $10^{20}-10^{21} \mathrm{~cm}^{-3}$ [9]. The time to take such doses is changed from 3.2 to 3200 years [9]. The main difficulty is the supply of laser radiation to the sensors in the working area of the reactor [9].

The application methods of Relaxed Optics for the receiving stable n-p junctions is expanded the technologic methods of modern optoelectronics. These methods may be applied for the other semiconductors, including $\mathrm{Si}, \mathrm{Ge}, \mathrm{GaAs}$, CdS, CdTe, ZnSe, $\mathrm{Hg}_{x} \mathrm{Cd}_{1-x} \mathrm{Te}$ and $\mathrm{Pb}_{x} \mathrm{Sn}_{1-x} \mathrm{Te}$.

Methods of RO may be used for the creation surface nanostructures, various volumetric devices and for the increasing of the resource of the existing optoelectronic devices, including semiconductor and solid laser, photodiodes, optical fibers, heterostructures and other.

\subsection{Processes of Reabsorption and Reirradiation and Its Possible Applications}

Simple examples of RO processes are the photochemical phenomena. The con- 
ditions of effectively of these processes may be divided on two groups [9].

First grope is including the case of light scattering on unstable or metastable centers. For this case basic role have the integral flux of radiation. These processes may be single-photon and cascade multiphoton. The $\mathrm{CO}_{2}$-laser annealing of ion-implanted layers $\mathrm{Mg}^{2+} / \mathrm{InSb}$ in pulse regime (duration of pulse 0.1 $\mu \mathrm{s}$ ) and stationary regime (duration of irradiation $2 \mathrm{~s}$ ) result to identical results: we have full crystallization of ion-implanted layer and activation of impurity. This process is example of processes of first group.

Second group processes are the processes of light scattering on stable centers. For this case basic role have intensity and time of irradiation. Laser implantation of InSb with help Ruby-laser irradiation is example of phenomena of second group.

Basic peculiarities of Relaxed optical phenomena may be analyzed with profiles of laser-induced subsurface donor centers in InSb (Figure 25 and Figure 26) [1] [6] [9] [41].

The profiles of the distribution the photostimulated donor centers in subsurface layers InSb are represented in Figure 26 [41]. The samples of p-type conductivity are irradiated by pulses of Ruby laser (wavelength $\lambda=0.69 \mu \mathrm{m}$, duration of pulse $\tau_{i}=20 \mathrm{~ns}$ ). For intensity of irradiation $I_{0}>0.001 \mathrm{~J} \cdot \mathrm{cm}^{-2}$ for InSb the n-layers on p-type materials are created. For intensity of irradiation $I_{0}<0.1$ $\mathrm{J} \cdot \mathrm{cm}^{-2}$ for InSb the profiles of the distribution of donor centers are represented the Buger-Lambert law (law of absorption the light in homogeneous media). For further increasing the irradiated intensity the profiles of the concentration donor centers have diffusion nature. The visible destruction of the irradiated semiconductor (melting, the change of the surface colour) had place for $I_{0}>0.3 \mathrm{~J} \cdot \mathrm{cm}^{-2}$ for InSb. For explanation of results of Figure 25 modified model of photoeffect

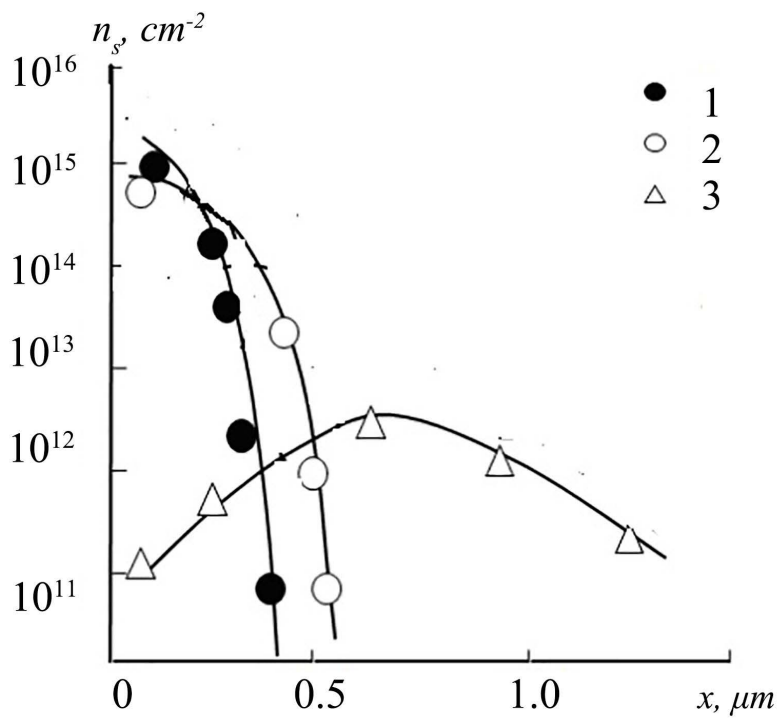

Figure 25. The profiles of the distribution the layer concentration of the donor centers in inverse layers InSb after Ruby laser irradiation with various density of energy (monoimpulse regime): 0.07 (1); $0.1(2) ; 0.16(3) \mathrm{J} \cdot \mathrm{cm}^{-2}$ [1] [6] [9]. 


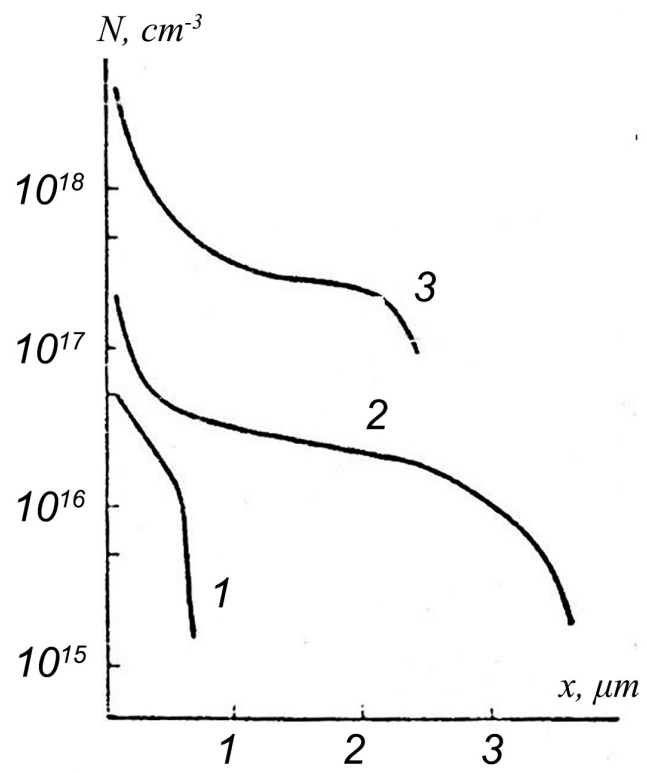

Figure 26. Profiles of the volume distribution electrons after laser irradiation. 1, 2: Ruby laser; 3: YAG:Nd laser. Energy density in pulse, $\mathrm{J} / \mathrm{cm}^{2}: 1$ - 5; 2 - 40 [41].

for irreversible case was created [1]. Curves 1,2 are corresponded to kinetic approximation of this model [1], curve 3 is corresponded to dynamic approximation of this model [1]. Microscopic mechanisms of these results was observed with help model of cascade step-by-step excitation of proper numbers and types of chemical bonds in the regime of saturation the excitation [1]. For indium antimonite two-dimensional lattice of sphalerite was used [1]. According to this model, curves 1, 2 of Figure 25 are corresponded to breakage two from three chemical bonds [1]. This case is corresponded of two-photonic absorption and may be represented as irreversible generation of second harmonic, observation of second harmonic for self-absorption range with help of optical and NLO methods is impossible [1].

The dependence of the creation donor centers in subsurface layers of InSb after Nd:YAG and Ruby laser irradiation is represented in Figure 26 [41]. The profiles were researched by V. Bogatyryov and G. Kachurin [41]. An irradiation was created with help Ruby laser $\left(\lambda=0.69 \mu \mathrm{m}, \tau_{i}=5-6 \mathrm{~ms}\right)$ and series of pulses Nd:YAG-laser $\left(\lambda=1.06 \mu \mathrm{m}, \tau_{i}=10 \mathrm{~ns}\right.$, frequency of repetition of pulses was 12.5 $\mathrm{Hz}$ ). A value of threshold the energy of creation $n$-layers is equaled $\sim 5 \mathrm{~J} / \mathrm{cm}^{2}$. A tendency of the saturation of the layer concentration had place for the energy density $\sim 40 \mathrm{~J} \cdot \mathrm{cm}^{-2}[41]$. These donor centers and proper damages are stable to temperature $400^{\circ} \mathrm{C}[40]$. The melting of surface has place for this value of the irradiation [41].

Form of curves 2 and 3 of Figure 26 showing an influence two- and multiphoton processes on formation of resulting profile of distribution of donor centers. Subsurface region $(\sim 0.5 \mu \mathrm{m})$ is corresponded to two-photonic self-absorption as for curves 1, 2 of Figure 25. Middle parts of curves 2 and 3 of Figure 26 are corresponded of multiphoton absorption with photon energy $0.18 \mathrm{eV}$ (band gap 
of InSb). Basic processes for this case are processes of photon fracturing and further reirradiation of bulk semiconductor [9]. Number of reradiations may be 400 - 500 [9]. Therefore "quantum yield" of creation donor centers for millisecond regime of irradiation is substantially smaller as for nanosecond regime.

Processes of very large laser pumping can cause suppression of oscillation and appearance of chaotization of laser radiation (Haken phenomenon) [1] [10].

All these processes were explained with one physical-chemical point of view, with using elementary energetic estimations [9]. The basic idea of this method is the successive saturation of excitation of proper chemical bonds of irradiated materials [9]. This method allows eliminating differences in the explanation of proper experimental data.

For the short regimes of irradiation, when irradiated time is less as relaxation time, the basic processes of irreversible changes in irradiated materials in the regime of saturation of excitations are straight processes of photoinization, including multiphoton processes of absorption. For indium antimonite most probable nonlinear processes for the regime of pulse Ruby-laser irradiation are the photon fragmentation and up-conversion absorption. First effect is basic for the excitation of first chemical bond (one photon break off $\sim 4-5$ bonds). This fact is caused grand relaxation time $\sim 10^{-7} \mathrm{~s}$. Up-conversion absorption is the result of the scattering Ruby-laser photons on excited electrons of first bond. This effect is caused break off second and third chemical bonds of InSb [1] [9]. For the irradiating time less as first relaxation time $\sim 10^{-7} \mathrm{~s}$ the processes of irradiated relaxation are negligible. But for the regimes of irradiation with time $\sim 10^{-3} \mathrm{~s}$ the processes of reirradiation have grand value on the processes of the formation irreversible changes in irradiated materials.

Higher concentration of donor centers for more short regimes of irradiation (Figure 26) is caused of processes of reradiation. For the regimes of irradiation with $\tau_{i} \gg \tau_{r 1}$ we have two types of irradiation: first order, basic, Ruby-laser irradiation with $h v=1.78 \mathrm{eV}$, and second order reradiation with $h v=E_{g}=0.18 \mathrm{eV}$ for InSb [9].

Rough estimation of effects of reemission may be made with help next formulas. The first part of reemission is equaled

$$
I_{r 1}=\frac{\tau_{r 1}}{\tau_{i}} I_{0} \mathrm{e}^{-\alpha x}
$$

Let this part of absorbed radiation is reemitted. In the next time the absorbed irradiation may be represented in the next form

$$
I_{r 2}=\frac{\tau_{r 1}}{\tau_{i}}\left(1-\frac{\tau_{r 1}}{\tau_{i}}\right) I_{0} \mathrm{e}^{-\alpha x}+\frac{\tau_{r 1}}{\tau_{i}}\left(1-\frac{\tau_{r 1}}{\tau_{i}}\right) I_{0} \mathrm{e}^{-\alpha_{1} x} I_{0} \mathrm{e}^{-\alpha_{2} x},
$$

where $\alpha_{1}$ : absorption factor of radiation with $h v=E_{g}$ (laser effect) and $\alpha_{2}$ : absorption factor of "blooming" radiation.

Second term in (56) is represented up-conversed absorption, which is caused irreversible changes in semiconductor. Second and third relaxation times are 
considerably greater as time of irradiation. Therefore second term in (56) may be represented as "irreversible" term. For the receiving number of reemissions $n$ we must multiply second term of formula (56) on $n$ and equate to intensity of saturation of excitation $I_{\text {sat }}$ Then

$$
n=\frac{\tau_{i} I_{\text {sat }}}{\tau_{r}\left(1-\frac{\tau_{r}}{\tau_{i}}\right) I_{0}} .
$$

After substitution proper value of $I_{\text {sat }}$ from [9] and $I_{0}$ from Figure 26 we have $n \cong 10 \div 500$.

It is very rough estimations. But experimental data of Figure 26 are certificated this hypothesis. Surface and volume concentrations donor centers in InSb after irradiation of nanosecond Ruby-laser pulses (Figure 25) is more in 3 - 4 orders as after millisecond irradiation (Figure 26).

For this case we can propose next simple model. The part of absorbed irradiation with including process of $n$-reirradiation may be represented as

$$
I_{n}=\frac{\tau_{r}}{\tau_{i}}\left(1-\frac{\tau_{r}}{\tau_{i}}\left(1-\frac{\tau_{r}}{\tau_{i}}\left(1-\frac{\tau_{r}}{\tau_{i}}\right) \cdots\right)\right) I_{0} \mathrm{e}^{-\alpha \chi} .
$$

After using of formula for geometrical progression this relation may be represented in next form [9]:

$$
I_{n}=\frac{\frac{\tau_{r}}{\tau_{i}}}{1+\frac{\tau_{r}}{\tau_{i}}} I_{0} \mathrm{e}^{-\alpha x} .
$$

with help formula (59) we can receive ratio for $\frac{\tau_{r}}{\tau_{i}}$ for curves 2 and 3 of Figure 26. In this case we can approximate $I_{n}$ as average value for tails of this curve and $I_{0} \mathrm{e}^{-\alpha x}$ as average value for their subsurface parts. For this case we have $\frac{\tau_{r}}{\tau_{i}} \approx 0.05-0.1$. For Ruby laser irradiation (curve 2 of Figure 26) we can determine relaxation time $\tau_{r} \approx(0.05-0.1) \tau_{i} \approx(0.25-0.5) \mathrm{ms}$. This time is equal zero for curve 1 of Figure 26 because processes of second-order reirradiation have not influence on the irreversible processes.

We can compare the efficiency of generation donor centers for the nanosecond (curve 2 of Figure 26) and millisecond (curve 2 of Figure 26) regimes of irradiation. Curve 2 of Figure 25 is represented pure irreversible process. Therefore, the comparative efficiency of using millisecond laser irradiation for creation of irreversible changes in irradiated matter may be determined with help next formula.

$$
\beta=\frac{\frac{I_{\text {sat }}}{N_{\text {avsat }}}}{\frac{I_{\text {ir }}}{N_{\text {avir }}}} .
$$


This value is equaled $2.5 \times 10^{-6}$ for subsurface part of curve 2 of Figure 26 and $2.5 \times 10^{-7}$ for tail part of curve 2 of Figure 26. For curve 1 of Figure 26 this efficiency is equaled $2 \times 10^{-6}$. Therefore, processes of reirradiation may be used for the formation more deep parts of irradiated matter [9].

Forms of profiles of distribution of donor centers are various too. The maximum of distribution is displaced in volume for nanosecond regime of irradiation. It is effect isn't characterized for millisecond regime of irradiation (Figure 26). Multipulse regime of irradiation of nanosecond Nd:YAG laser is analogous to Ruby-laser in millisecond regime, but for this case we have more discrete process of irradiation. The reemission is caused the decreasing concentrations of donor centers and increasing the depth of donor layers.

Effect of reirradiation in $\mathrm{RO}$ is analogous to famous G. Gamov Urca-process [9] and therefore may be called as optical Urca-process: more part of absorptive and reabsorptive energy is radiated and reradiated and cause not the phase transformations in irradiated matter.

The honeycomb model of laser annealing [19] may be realized in this case too.

These processes were named laser implantation [1] [9]. It may be used for creation off new technologies of optical and electronic devices. The problems of a creation of the three-dimensional periodical electronic structures are very important and may be having good future.

With help of these processes we can correct proper properties of materials and devices. High thermal stability of receiving donor centers on indium antimonite $\left(350^{\circ} \mathrm{C}-400^{\circ} \mathrm{C}\right)$ may be allowed to refine the basic characteristics photoelectronic infrared devices. But for this we must select correct regime of irradiation.

This method allows receiving materials with properties, which can't be received with help other methods. Therefore, using of this method is expanded fundamental and applied aspects of modern laser physics and optoelectronics.

So, layers of Figure 26 may be used for the receiving $n^{+}-n-p$ junctions, layers of Figure 25 for the creation n-p junctions (curves 2 and 3 of Figure 24) [1] [9].

\subsection{Other Applications}

Surface periodical structures may be used as diffractive lattices [9]. It was basic cause of research A. Bonch-Bruevich and his colleague's [4].

Multistructural nanohills (Figure 1) and microcolumns (Figure 2) may be used for the creation new elements of optoelectronics, including nanoelectronics (diodes matrixes, filter matrixes etc.).

Laser technology may be used for the selection various crystal structures of metals on the example of titanium (Figures 3-5). After laser irradiation hexagonal titanium is localized in subsurface regions, cubic in volume of irradiated material.

Possible applications of phenomena and processes of laser-induced breakdown of matter may be distributed on two groups: positive and negative. 
Negative processes are connected with laser-induced destruction of corresponding elements of optoelectronic systems and decreasing of its lifetime. Therefore, in this case we must use only low intensity irradiation and unfocused irradiation. These procedures must be included in work regimes of fiber optic communication lines and other devices of modern photonics.

Positive application may be next. Well known the using laser irradiation for the laser-annealing of ion-implanted layers of semiconductors [1] [6] and for the increasing the lifetime of magnetic sensors and other elements of nuclear reactors [9]. In this case we have low intensity of irradiation of light scattering on unstable or metastable centers, basically photochemical processes.

Next stage of possible applications laser-induced, including optical breakdown, processes is next. Focused laser irradiation may be used as source of diffraction stratification of initial laser irradiation, a generation of Cherenkov radiation, interference of this Cherenkov radiation and the creation cascade of destruction, which can use as volume diffractive lattices or other elements of controlling the laser irradiation and as elements of multifunctional heterostructures (Figure 19 and Figure 20). These multifunctional structures may be used as passive and active elements with various system values. Roughly speaking it may be semireproducing devices.

The laser-induced optical breakdown of matter may be used for the change functional properties of optoelectronic devices and elements and for the creation new systems.

In more general case the methods of Relaxed and Nonlinear Optics may be used for the creation new elements of optoelectronic systems with nonequilibrium and irreversible properties [1] [6] [9].

It may be used for the creation new circle elements and devices and expansions of functional activity of existing devices.

The laser-induced optical breakdown of air may be used for energy transmission over long distances [4] [7] [37].

The problem of the irreversible interaction light and matter have large influence on the life time the objectives of art (painting, buildings, monuments and other) [1].

Two aspects of this problem are represented. First, natural: in this case the light is the source of the aging and the destruction. Second, creational: the light is the source of the creation and the renewal. The union of the both approaches may be used for the decoding the ancient objectives of the culture.

These ways of the application the relaxed optical phenomena are discussed.

The problem of the aging the objectives of the art is very ancient. The basic objectives of art and archaeology are meant in time. The light is one of the basic causes of this meaning at the direct and derivative applications. The wind, water, temperature and light are the basic parameters of the destruction, renewal and creation in the art, archaeology and architecture.

The one of the more ancient the cultural monuments are created in stones, 
ceramics, papers and metals. Its lived the thousands years. The other monuments (which is built on other materials: tree, loam, sand, reed, paint) have more short time of the life (same centuries and less). Two problems must be resolved in this region of human activity. First, the creation the monuments of art with long time of the life, and second, the decoding and renewal the ancient exemplars of art and culture.

First are the problem of the present and the future of the culture and second is the problem of the past of the culture. But both these problems may be united in one more general. The ways of the resolution this problem as whole is next: first, the theoretical researches in the modeling new methods of the representation and the creation of the cultural objects; second, the theoretical and physical-chemical researches in the region of the decoding and renewal of the ancient cultural objects. The first direction is more rich and intensive than second because one is used the basic achievement of the modern science and technology.

Laser-induced micro-holes have a broad application in different manufacturing sectors, such as microelectronics, aeronautics, electric engineering, medicine and many others. Creation of micro-holes with given parameters simplifies application of new generation of microelectronic components, microsensors, filters, surgical instruments etc. Therefore, micro-hole drilling in a wide range of materials, such as steel, copper, aluminum, etc., has received much attention [42] [43] [44] [45].

The demands for high precise micro-holes are increasing with the increasing of application range, therefore traditional mechanical drilling is slightly applicable. On the other hand, laser drilling using continuous-wave lasers is accompanied with the formation of melting layer and due to its uncontrolled redeposition recast layer. The best way of overcoming main laser drilling disadvantages is laser pulse shortening, so that only material ablation by nonlinear absorption takes place [42]. Such material ablation could be achieved by using of picosecond or even femtosecond laser sources because ablation of pulsed laser radiation depends on the length of the respective pulse. Different pulse lengths have different etching mechanisms during removal of the material, and the shape of the material surface will be different after the removal process.

As shown in Figure 27(a) [45], the long-pulse ablation area is much larger than the laser focused spot, which can easily lead to material melting, and the debris of microcracks and splattering processing will pollute the processing area.

Other adverse effects include the damage to nearby structures and the area around the ablated area becomes wrinkled. As shown in Figure 27(b) [45], compared with the long-pulse laser, the ultrashort pulse laser has the advantages of very short pulse duration, high pulse peak power, thermal effects and shock wave can be avoided, accurate targeting focused positioning and so on. Structures manufactured by femtosecond laser have extremely sharp edges compared to long pulses.

Although studies on micro-hole drilling by short pulsed lasers have received much attention, drilling of high precise micro-holes with minimal or no thermal damage still remains a major challenge [42] [43] [44] [45]. 
In work [42] micro-hole drilling was processed using TRUMPF TruMicro 5000 picosecond laser. The wavelength of the laser was $515 \mathrm{~nm}$ with a pulse width of $10 \mathrm{ps}$ at $400 \mathrm{kHz}$ pulse repetition frequency. The power density was $5.72 \times 10^{5} \mathrm{~W} \cdot \mathrm{cm}^{-2}$, and equivalence laser fluence was $1.43 \mathrm{~J} \cdot \mathrm{cm}^{-2}$. Micro-holes were received in steel SAE-304 (Figure 28) [42]

Line profiles of drilled micro-holes for different amounts of shots are represented on Figure 29 [42].

Depth of the hole drilled using picosecond laser for different numbers of pulses was researched too (Figure 30) [42].

As seen from Figure 30 the dependence of the depth of holes tends to saturate. This is obviously due to the increase in light reflection compared to previous pulses.

In order to avoid dross (Figure 28), it is advisable to choose the modes of laser irradiation such that the main processes are sublimation of the irradiated material. This mode is easy to choose, since the binding energy of near-surface atoms is 1.2 - 2 times less than that of bulk atoms, while we will have laser-induced sputtering with a solid rather than a liquid phase.

(a)
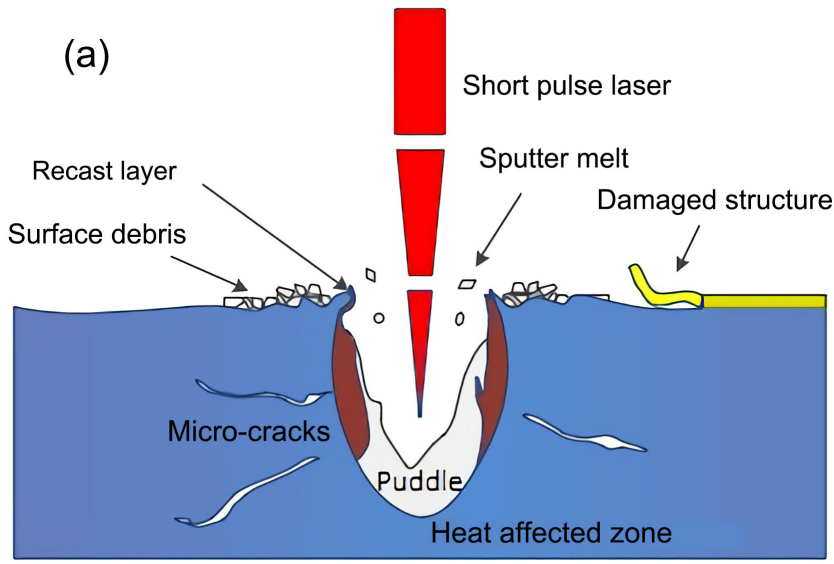

(b)

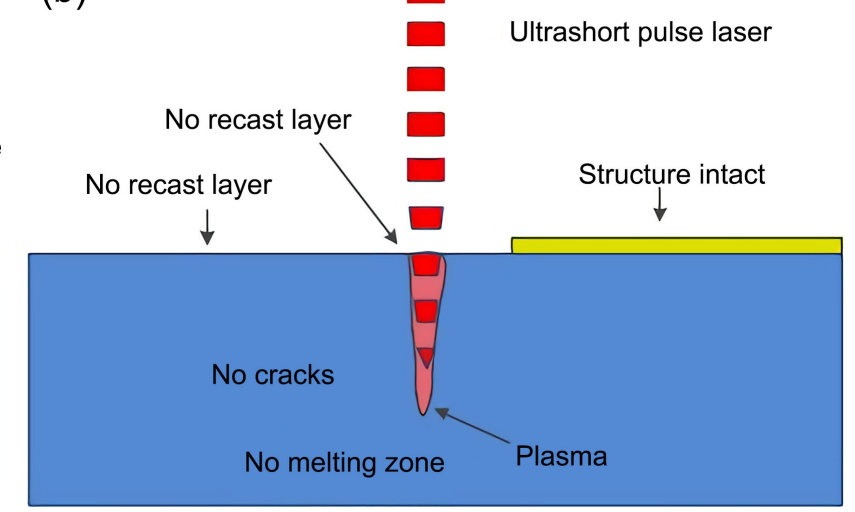

Figure 27. Physical phenomena of irradiation by short laser pulse (a), ultrashort laser pulse (b).

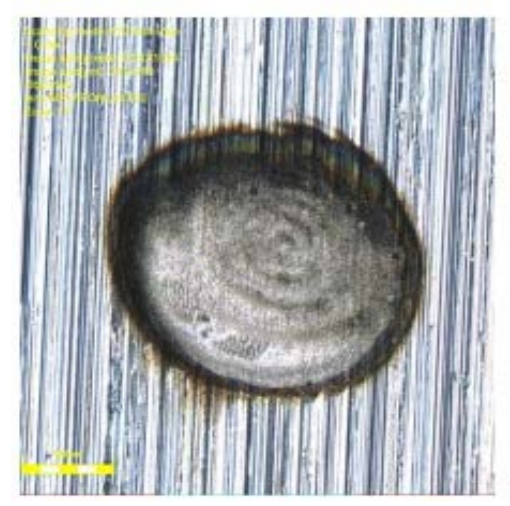

(a)

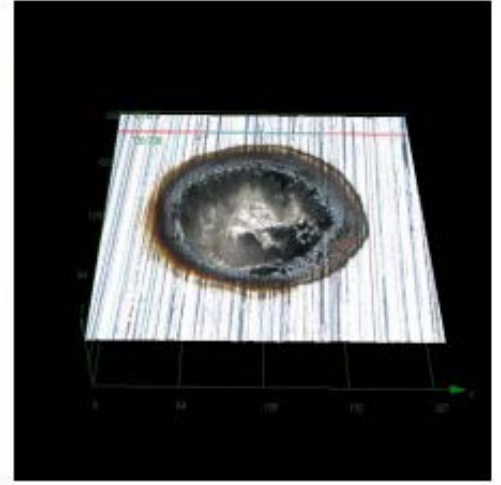

(b)

Figure 28. Micro-holes drilled in stainless steel using picosecond laser: (a) 30 shots, 2D view; (b) 190 shots, 3D view. 


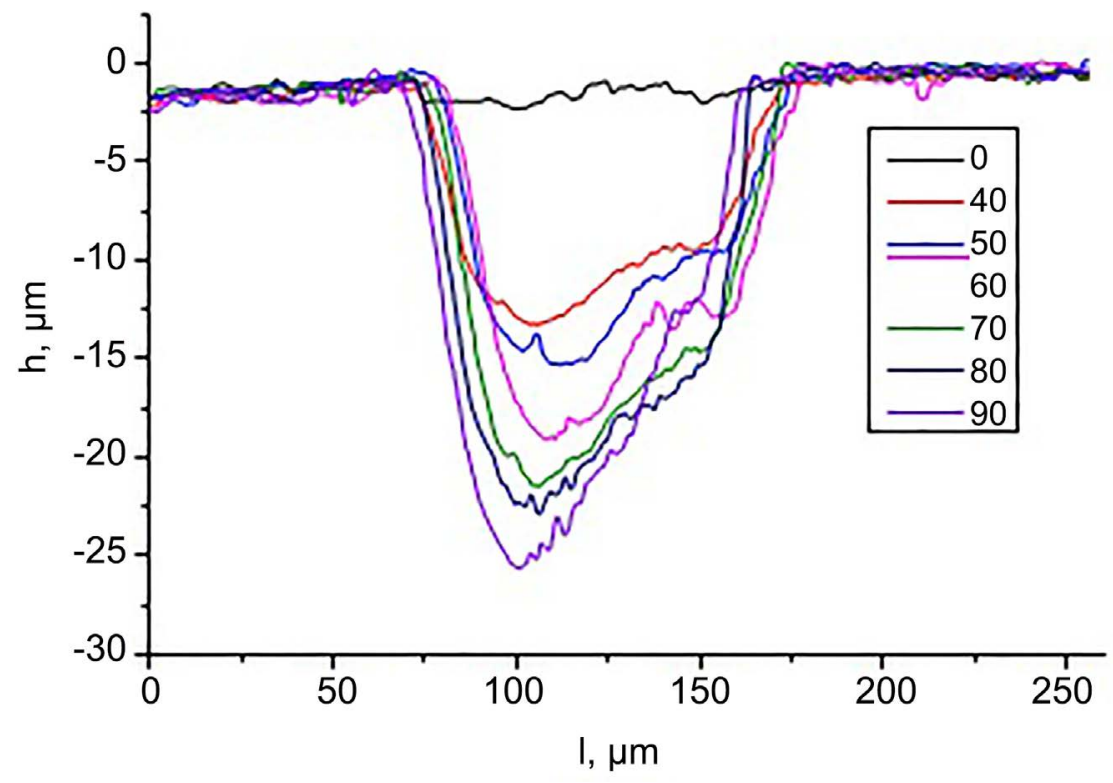

Figure 29. Line profiles of drilled micro-holes for different amounts of shots [42].

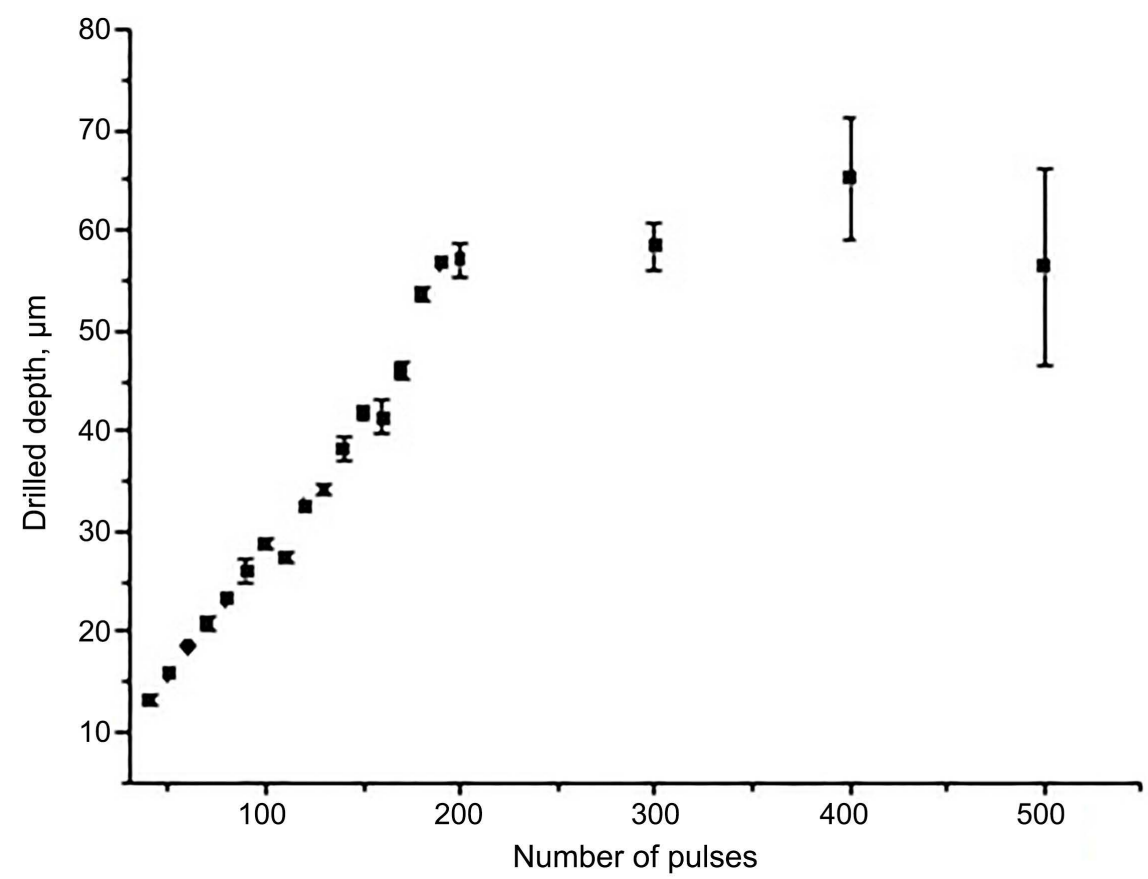

Figure 30. Depth of the hole drilled using picosecond laser for different numbers of pulses [42].

The edges of the holes will be smoother and by adjusting the irradiation time, we can get holes in a wider range of sizes. For these purposes, longer irradiation regimes can be used.

An interchange of Relaxed and Nonlinear optical processes may be used for the creation new devices for the work in aggressive and hard-to-reach media, including chemical and nuclear industries, cosmos, and ocean and for the creation new chapters of laser technologies [4] [6]. 


\section{Conclusions}

1) The question of the need to create relaxation optics designed to explain the phenomena that arise in laser technologies is analyzed.

2) Surface phenomena of Relaxed Optics are considered. At the same time, attention is focused on photostimulated phase transformations in laser-irradiated materials. Cascade model of step-by-step of proper chemical bonds in the regime of saturation of excitation is used.

3) Short review of volume processes of RO is represented, too. Role of few Nonlinear Optical processes (electrostriction, Brillouin scattering a.o.) in the formation of proper irreversible changes is shown.

4) Processes of creation of laser-induced filaments and optical breakdown are explained with unitary point of view. Complex cascade model (focusing, diffractive stratification, generation of the surface optical-induced Cherenkov radiation, interference of this Cherenkov radiation and generation of electromagnetic shock processes) allow to explain main experimental data.

5) Applications of laser radiation for the annealing surface damages of ion-implantation and volume damages after neutron irradiation are discussed.

6) New area of processes of the reabsorption and reirradiation as technological methods of modern optoelectronics are represented.

7) Possible applications of method of RO in painting, architecture and building are analysed.

8) Some questions of using laser irradiation in metallurgy and material science are discussed, too.

\section{Acknowledgements}

Author wishes to thank M. Romanyuk, O. Vlokh, Ya. Dovhyy, L. Laz'ko, A. Tsaryk, V. Peretyatko, V. Antonov-Romanovskiy, I. Frank, P. Cherenkov, V. Makin, A. Medvid', I. Stoyanova, V. Bonch-Bruyevich, Yu. Klimontovich, G. Kachurin, V. Stafeev, M. Makoviychuck, S. Vorobyov, F. Zaitov, M. Avdekovich, V. Shyrokovskiy, A. Svidzinskyy, P. Danyl'chenko, A. Frimer, V. Heyfets, V. Cheltsov and V. Holovatskiy for the discussion of basic idea of my research and L. Maidanovich-Sorensen, M. Shevchuk (Maydanovych), D. Shvalikovskyy and Yu. Fedonyuk for help in the preparation of this paper.

\section{Conflicts of Interest}

The author declares no conflicts of interest regarding the publication of this paper.

\section{References}

[1] Trokhimchuck, P. (2016) Relaxed Optics: Realities and Perspectives. Lambert Academic Publishing, Saarbrukken.

[2] Shen, Y.R. (1984) The Principles of Nonlinear Optics. John Wiley \& Sons, New York. 
[3] Trokhimchuck, P. (2013) Nonlinear and Relaxed Optical Processes: Problems of Interactions. Vezha-Print, Lutsk.

[4] Veyko, V.P., Libenson, M.N., Chervyakov, G.G. and Yakovlev, E.B. (2008) Interaction of Laser Radiation with Matter. Power Optics. Phyzmatlit, Moscow. (In Russian)

[5] Newton, I. (1704) Opticks: Or, a Treatise of the Reflexions, Refractions, Inflexions and Colours of Light. Also to Treatises of the Species and Magnitude of Curvilinear Figures. Printed for the Royal Society. https://doi.org/10.5479/sil.302475.39088000644674

[6] Trokhimchuck, P. (2011) Foundations of Relaxed Optics. Vezha-Print, Lutsk.

[7] Trokhimchuck, P. (2020) Optical Breakdown in Matter: Retrospective and Perspective. In: Advanced in Engineering Technology, Vol. 3, Ch. 7, AkiNik Publications, New Delhi, 101-132.

[8] Trokhimchuck, P. (2020) Role Physical-Chemical Processes in the Generation of Laser-Induced Structures. In: Research Trends in Chemical Sciences, Vol. 11, Ch. 6, AkiNik Publications, New Delhi, 109-140.

[9] Trokhimchuck, P. (2020) Relaxed Optics: Modeling and Discussions. Lambert Academic Publishing, Saarbrukken.

[10] Haken, H. (2004) Synergetics: Introduction and Advanced Topics. Springer, Berlin. https://doi.org/10.1007/978-3-662-10184-1

[11] Lax, M. (1960) Cascade Capture of Electrons in Solids. Physical Review, 119, 1502-1523. https://doi.org/10.1103/PhysRev.119.1502

[12] Lax, M. (1952) Frank-Condon Principle and Its Application to Crystals. The Journal of Chemical Physics, 20, 1752-1763. https://doi.org/10.1063/1.1700283

[13] Medvid, A. (2010) Nano-Cones Formed on a Surface of Semiconductors by Laser Radiation: Technology, Model and Properties. In: Lupu, N., Ed., Nanowires Science and Technology, Inech, Vukovar, 61-82. https://doi.org/10.5772/39493

[14] Pedraza, A.J., Fowlkes, J.D. and Lowndes, D.H. (1999) Silicon Microcolumn Arrays Growth by Nanosecond Pulse Laser Irradiation. Applied Physics Letters, 74, 2222-2224. https://doi.org/10.1063/1.123838

[15] Pedraza, A.J., Guan, Y.F., Fowlkes, J.D., Smith, D.A. and Lowndes, D.H. (2004) Nanostructures Produced by Ultraviolet Laser Irradiation of Silicon. I. Rippled Structures. Journal of Vacuum Science \& Technology B, 22, 2823-2835.

https://doi.org/10.1116/1.1821575

[16] Shen, M., Carey, J.E., Crouch, C.H., Kandyla, M., Stone, H.A. and Mazur, E. (2008) High-Density Regular Arrays of Nano-Scale Rods Formed on Silicon Surfaces via Femtosecond Laser Irradiation in Water. Nanoletters, 8, 2087-2091. https://doi.org/10.1021/nl080291q

[17] Makin, V.S. (2013) Peculiarities of the Formation the Ordered Micro and Nanostructures in Condensed Matter after Laser Excitation of Surface Polaritons Modes. D.Sc. Thesis, State University of Information Technologies, Mechanics and Optics, Saint-Petersburg. (In Russian)

[18] Tsukamoto, M., Asuka, K., Nakano, N., Hashida, M., Ratto, M., Abe, N. and Fujita, M. (2006) Period Microstructures Produced by Femtosecond Laser Irradiation on Titanium Plate. Vacuum, 80, 1346-1350. https://doi.org/10.1016/j.vacuum.2006.01.016

[19] Philips, J.C. (1981) Metastable Honeycomb Model of Laser Annealing. Journal of Applied Physics, 52, 7397-7402. https://doi.org/10.1063/1.328729 
[20] Trokhimchuck, P.P. (2007) Radiation Physics of Status Solid. Vezha, Lutsk, 394. (In Ukrainian)

[21] Trokhimchuck, P. (2021) Thomson-Benard Phenomena and Relaxed Optics. IJARPS, 8, 1-15.

[22] Rayleygh (1916) On Convective Currents in a Horizontal Layer of Fluid Then the Higher Temperature Is on the Underside. The Philosophers Magazine, 32, 529-546.

[23] Chandrasekar, S. (1961) Hydrodynamic and Hydromagnetic Stability. Dover Publications, New York.

[24] Sharma, B.S. (1968) Laser-Induced Dielectric Breakdown and Mechanical Damage in Silicate Glasses. Ph.D. Thesis, Simon Fraser University Press, Burnaby.

[25] Manenkov, A.A. and Prokhorov, A.M. (1986) Laser Destruction of Radiolucent Solid Bodies. Progress of Physical Science, 148, 179-211. (In Russian)

https://doi.org/10.3367/UFNr.0148.198601h.0179

[26] Stuart, B.S., Feit, M.D., Rubenchik, A.M., Shore, B.W. and Perry, M.D. (1995) Laser-Induced Damage in Dielectrics with Nanosecond to Subpicosecond Pulses. Physical Review Letters, 74, 2248-2252. https://doi.org/10.1103/PhysRevLett.74.2248

[27] Kroll, N. and Watson, K.M. (1972) Theoretical Study of Ionization of Air by Intense Laser Pulses. Physical Review A, 5, 1883-1905.

https://doi.org/10.1103/PhysRevA.5.1883

[28] Minardi, S., Gopal, A., Tatarakis, M., Couairon, A., Tamosăuskas, G., Piskarskas, R., Dubietis, A. and Di Trapani, P. (2008) Time-Resolved Refractive Index and Absorption Mapping of Light-Plasma Filaments in Water. Optics Letters, 33, 86-88. https://doi.org/10.1364/OL.33.000086

[29] Okada, T., Tomita, T., Matsuo, S., Hashimoto, S., Ishida, Y., Kiyama, S. and Takahashi, T. (2009) Formation of Periodic Strain Layers Associated with Nanovoids inside a Silicon Carbide Single Crystal Induced by Femtosecond Laser Irradiation. Journal of Applied Physics, 106, Article ID: 054307. https://doi.org/10.1063/1.3211311

[30] Okada, T., Tomita, T., Matsuo, S., Hashimoto, S., Kashino, R. and Ito, T. (2012) Formation of Nanovoids in Femtosecond Laser Irradiated Single Crystal Silicon Carbide. Material Science Forum, 725, 19-22. https://doi.org/10.4028/www.scientific.net/MSF.725.19

[31] Yablonovich, E. (1971) Optical Dielectric Strength of Alkali Halide Crystals Obtained by Laser Induced Breakdown. Applied Physics Letters, 19, 495-497. https://doi.org/10.1063/1.1653788

[32] Berge, L., Skupin, S., Lederer, F., Mejean, G., Yu, J., Kasparian, J., Salmon, E., Wolf, J.P., Rodrigues, M., Wöste, L., Bourayou, R. and Saurbrey, P. (2004) Multiple Filamentation of Terawatt Laser in Air. Physical Review Letters, 92, Article ID: 225002. https://doi.org/10.1103/PhysRevLett.92.225002

[33] Wu, J., Zhao, J., Qiao, H., Hu, X. and Yang, Y. (2020) The New Technologies Developed from Laser Shock Processing. Material(Basel), 13, 1453-1456. https://doi.org/10.3390/ma13061453

[34] Golub, I. (1990) Optical Characteristics of Supercontinuum Generation. Optics Letters, 15, 305-307. https://doi.org/10.1364/OL.15.000305

[35] Frank, I.M. (1988) Cherenkov Radiation. Theoretical Aspects. Nauka, Moscow. (In Russian)

[36] Bohr, N. (1950) The Passage of Charged Particles through Matter. IL, Moscow. (In 
Russian)

[37] Boyd, R.W., Lukishova, S.G. and Shen, Y.-R. (2009) Self-Focusing: Past and Present. Springer Series: Topics in Applied Physics, Vol. 114, Springer, Berlin.

[38] Rayleygh (1917) On the Pressure Developed in a Liquid during the Collapse of a Spherical Cavity. The London, Edinburgh and Dublin Philosophical Magazine and Journal of Science, 34, 94-97. https://doi.org/10.1080/14786440808635681

[39] Lauteborn, W. and Kurz, T. (2010) Physics of Bubble Oscillations. Reports on Progress in Physics, 77, Article ID: 106501. https://doi.org/10.1088/0034-4885/73/10/106501

[40] Khaybullin, I.B. and Smirnov, L.S. (1985) Impulse Annealing of Semiconductors. The State of the Problem and Unresolved Questions (Review). Physics and Technics of Semiconductors, 19, 569-589. (In Russian)

[41] Bogatyryov, V.A. and Kachurin, G.A. (1977) The Creation Low Resistively n-Layers on InSb with Help the Impulse Laser Irradiation. Physics and Technics of Semiconductors, 11, 100-102. (In Russian).

[42] Dupliak, I., Li, F.P. and Guang, F. (2019) Micro-Hole Drilling of Stainless Steel Using Short Pulse Laser. Proceedings Third International Conference "Actual Problems of Fundamental Science", Lutsk, 1-5 June 2019, 42-44.

[43] Choi, W., Kim, H.Y., Jeon, J.W., Chang, W.S. and Cho, S.-H. (2017) Vibration-Assisted Femtosecond Laser Drilling with Controllable Taper Angles for AMOLED Fine Metal Mask Fabrication. Materials, 10, 212. https://doi.org/10.3390/ma10020212

[44] Jahnsa, D., Kaszemeikata, T., Muellera, N., Ashkenasia, D., Dietricha, R. and Eichlera, H.J. (2013) Laser Trepanning of Stainless Steel. Physics Procedia, 41, 630-635. https://doi.org/10.1016/j.phpro.2013.03.126

[45] Leitz, K.H., Redlingshöfer, B., Reg, Y., Otto, A. and Schmidt, M. (2011) Metal Ablation with Short and Ultrashort Laser Pulses. Physics Procedia, 12, 230-238.

https://doi.org/10.1016/j.phpro.2011.03.128 